\title{
Transgenic Monkey Model of the Polyglutamine Diseases Recapitulating Progressive Neurological Symptoms
}

\author{
Ikuo Tomioka, ${ }^{1}$ Hidetoshi Ishibashi, ${ }^{1}$ Eiko N. Minakawa, ${ }^{2}$ Hideyuki H. Motohashi, ${ }^{1}$ Osamu Takayama, ${ }^{1}$ \\ (1) Yuko Saito, ${ }^{3}$ H. Akiko Popiel, ${ }^{2}$ Sandra Puentes, ${ }^{1}$ Kensuke Owari, ${ }^{1}$ Terumi Nakatani, ${ }^{1}$ Naotake \\ Nogami, ${ }^{1}$ Kazuhiro Yamamoto, ${ }^{4}$ (D) Satoru Noguchi, ${ }^{5}$ Takahiro Yonekawa, ${ }^{5}$ Yoko Tanaka, ${ }^{3}$ Naoko Fujita, ${ }^{1}$ \\ Hikaru Suzuki, ${ }^{1}{ }^{-}$Hisae Kikuchi, ${ }^{2}$ Shu Aizawa, ${ }^{2}$ Seiichi Nagano, ${ }^{6}$ Daisuke Yamada, ${ }^{2}$ Ichizo Nishino, ${ }^{5}$ \\ DNoritaka Ichinohe, ${ }^{7}$ Keiji Wada, ${ }^{2}$ Shinichi Kohsaka, ${ }^{8}$ (D) Yoshitaka Nagai, ${ }^{2}$ and ${ }^{\text {DKazuhiko Seki }}{ }^{1}$
}

DOI:http://dx.doi.org/10.1523/ENEURO.0250-16.2017

\begin{abstract}
${ }^{1}$ Department of Neurophysiology, National Institute of Neuroscience National Center of Neurology and Psychiatry, Tokyo 187-8502, Japan, ${ }^{2}$ Department of Degenerative Neurological Diseases, National Institute of Neuroscience National Center of Neurology and Psychiatry, Tokyo 187-8502, Japan, ${ }^{3}$ Department of Pathology and Laboratory Medicine, National Institute of Neuroscience National Center of Neurology and Psychiatry, Tokyo 187-8502, Japan, ${ }^{4}$ Division of Laboratory Animals Resources, National Institute of Neuroscience National Center of Neurology and Psychiatry, Tokyo 187-8502, Japan, ${ }^{5}$ Department of Neuromuscular Research, National Institute of Neuroscience National Center of Neurology and Psychiatry, Tokyo 187-8502, Japan, ${ }^{6}$ Department of Peripheral Nervous System Research, National Institute of Neuroscience National Center of Neurology and Psychiatry, Tokyo 187-8502, Japan, ${ }^{7}$ Department of Ultrastructural Research, National Institute of Neuroscience National Center of Neurology and Psychiatry, Tokyo 187-8502, Japan, ${ }^{8}$ Department of Neurochemistry, National Institute of Neuroscience National Center of Neurology and Psychiatry, Tokyo 187-8502, Japan
\end{abstract}

\begin{abstract}
Age-associated neurodegenerative diseases, such as Alzheimer's disease, Parkinson's disease, and the polyglutamine (polyQ) diseases, are becoming prevalent as a consequence of elongation of the human lifespan. Although various rodent models have been developed to study and overcome these diseases, they have limitations in their translational research utility owing to differences from humans in brain structure and function and in drug
\end{abstract}

\section{Significance Statement}

Various neurodegenerative diseases, including Alzheimer's disease, Parkinson's disease, and the polyglutamine diseases, share a common pathomechanism via the abnormal accumulation of misfolded proteins in the central nervous system. Here, we have successfully established a transgenic marmoset model of the polyglutamine diseases, which recapitulates the human disease process including the accumulation of misfolded proteins and neuronal cell loss, resulting in neurologic symptoms. Because the neurologic symptoms initiated at 3-4 months after birth followed by gradual progression, our model opens a time window in future translational research to develop clinically relevant biomarkers and evaluate the preclinical efficacy of therapeutic candidates, making the most of the similarity of both brain structure and function, as well as drug metabolism, between humans and primates.

Received August 22, 2016; accepted March 7, 2017; First published March 28, 2017.

The authors declare no competing financial interests.
Author contributions: I.T., K.Y., K.W., S.K., Y.N., and K.S. designed research; I.T., H.I., E.N.M., H.H.M., O.T., Y.S., H.A.P., S.P., K.O., T.N., N.N., K.Y., S. Noguchi, T.Y., Y.T., N.F., H.S., H.K., S.A., S. Nagano, D.Y., and N.I. performed 
metabolism. Here, we generated a transgenic marmoset model of the polyQ diseases, showing progressive neurological symptoms including motor impairment. Seven transgenic marmosets were produced by lentiviral introduction of the human ataxin 3 gene with 120 CAG repeats encoding an expanded polyQ stretch. Although all offspring showed no neurological symptoms at birth, three marmosets with higher transgene expression developed neurological symptoms of varying degrees at 3-4 months after birth, followed by gradual decreases in body weight gain, spontaneous activity, and grip strength, indicating time-dependent disease progression. Pathological examinations revealed neurodegeneration and intranuclear poly $Q$ protein inclusions accompanied by gliosis, which recapitulate the neuropathological features of polyQ disease patients. Consistent with neuronal loss in the cerebellum, brain $\mathrm{MRI}$ analyses in one living symptomatic marmoset detected enlargement of the fourth ventricle, which suggests cerebellar atrophy. Notably, successful germline transgene transmission was confirmed in the secondgeneration offspring derived from the symptomatic transgenic marmoset gamete. Because the accumulation of abnormal proteins is a shared pathomechanism among various neurodegenerative diseases, we suggest that this new marmoset model will contribute toward elucidating the pathomechanisms of and developing clinically applicable therapies for neurodegenerative diseases.

Key words: Neurodegenerative disease; Polyglutamine disease; Transgenic monkey; Common marmoset

research; I.T. contributed unpublished reagents/analytic tools; I.T., H.I., E.N.M., H.H.M., O.T., Y.S., H.A.P., S.P., K.O., T.N., K.Y., S. Noguchi, T.Y., N.F., S.A., I.N., Y.N., and K.S. analyzed data; I.T., H.I., E.N.M., Y.S., H.A.P., S. Noguchi, Y.N., and K.S. wrote the paper.

This work was supported in part by Intramural Research Grants for Neurological and Psychiatric Disorders from the National Center of Neurology and Psychiatry [23-9, 26-11 to K.S., Y.N., and I.T.]; a Health Labour Sciences Research Grant for Research on Development of New Drugs from the Ministry of Health, Labour and Welfare, Japan and the Japan Agency for Medical Research and Development [26-005 to K.S., Y.N., and I.T.]; Grants-in-Aid for Challenging Exploratory Research from the Japan Society for the Promotion of Science, Japan [26670446 to Y.N.]; Grants-in-Aid for Young Scientists (B) from the Japan Society for the Promotion of Science, Japan [26870884 to I.T.]; a grant from Core Research for Evolutional Science and Technology (CREST) of the Japan Science and Technology Agency to Y.N; a research grant from the ALS "Inochi no Iro" Foundation to I.T.; and a research grant from the Takeda Science Foundation to I.T.

Ikuo Tomioka's present address: Institute for Biomedical Science, Interdisciplinary Cluster for Cutting Edge Research, Shinshu University, Nagano 3994598, Japan; Hidetoshi Ishibashi's present address: Animal Research Center, Tokyo Medical University, Tokyo 160-8402, Japan; Hideyuki H. Motohashi's and Osamu Takayama's present address: Assisted Reproductive Technology Center, Okayama University, Okayama 700-8530, Japan; Sandra Puentes's present address: Faculty of Engineering, Information and Systems University of Tsukuba, Ibaraki 305-8577, Japan; Naoko Fujita's present address: Division of Molecular Genetics, Institute for Comprehensive Medical Science, Fujita Health University, Aichi 470-1192, Japan; Yoshitaka Nagai's present address: Department of Neurotherapeutics, Osaka University Graduate School of Medicine, Osaka 565-0871, Japan.

\section{Introduction}

Modeling human diseases in experimental animals via genetic engineering is an indispensable step in studying the pathomechanisms of and developing therapies for intractable neurodegenerative diseases such as Alzheimer's disease (AD), Parkinson's disease (PD), Huntington's disease (HD), and the spinocerebellar ataxias (SCAs). Indeed, various transgenic and knockout/knockin mouse models of these neurodegenerative diseases have been developed and have substantially contributed to the understanding of basic disease mechanisms (Wong et al. 2002; Rockenstein et al. 2007). However, rodent models have limitations in translational research in terms of evaluating the therapeutic efficacy and metabolic profiles of drug candidates, as well as in developing disease biomarkers, mainly because of the differences in metabolism between humans and rodents (Chan, 2013; Kishi et al. 2014). Furthermore, differences between humans and rodents in the structure and physiologic functions of the brain have resulted in difficulties in reproducing the selective vulnerability of specific neurons or circuits in these mouse models (Chan, 2013; Kishi et al. 2014). In addition, the small-sized brains of rodents are difficult to be analyzed anatomically or functionally in detail by in vivo imaging techniques such as MRI or positron emission tomography (PET). These limitations have resulted in the failure to predict the efficacy of clinical trials in human patients from the experimental findings obtained from rodent models of neurodegenerative diseases, lessening the preclinical value of rodent models (Doody et al. 2013, 2014; Salloway et al. 2014). Therefore, the establishment of clinically relevant models of neurodegenerative diseases using nonhuman primates is imperative for accelerating our understanding of pathophysiological mechanisms and the development of clinically applicable therapies (Morton and Howland, 2013; Howland and Munoz-Sanjuan, 2014).

Various nonhuman primate models of human diseases using the chimpanzee, rhesus macaque, cynomolgus macaque, common marmoset, or common squirrel monkey have been reported (Chan, 2013). Among them, the marmoset, a small, nonendangered nonhuman primate, offers many advantages regarding its reproductive features and small body size. Marmosets routinely ovulate multiple oocytes per ovarian cycle, have a short gestation period, and reach sexual maturity at $\sim 1$ year of age (Kishi et al. 2014). Their small body size also enables us to handle them with ease, which translates into lower caging and feeding costs. Furthermore, reproductive technologies for the marmoset, such as embryonic stem cells (Sasaki et al. 2005), transgenic animals with transgene germline transmission (Sasaki et al. 2009), and induced pluripotent stem cells (Tomioka et al. 2010), have been rapidly developed in just the past decade. These advantages of the marmoset compared with other nonhuman primates may provide better outcomes once it has been used in translational research.

In this study, we successfully generated a marmoset model of the polyglutamine (polyQ) diseases, which are a group of inherited neurodegenerative diseases including $\mathrm{HD}$ and various types of SCA. The polyQ diseases are 
caused in common by an expansion mutation of the polymorphic CAG repeat ( $>35-40$ repeats) encoding the glutamine stretch in each disease-causative gene. One of the distinctive features of the polyQ diseases is a tight genotype-phenotype correlation between the number of CAG repeats and the age of disease onset, which makes it a promising avenue to establish a symptomatic transgenic marmoset model by genetic engineering. Considering that various neurodegenerative diseases including AD, $\mathrm{PD}$, and the polyQ diseases share a common pathomechanism of abnormal accumulation of misfolded proteins (Nagai and Popiel, 2008; Costa and Paulson, 2012), our success in the establishment of a symptomatic transgenic marmoset line accompanied by abnormal protein inclusions and neurodegeneration opens new avenues toward elucidating the pathomechanism of and developing therapeutic approaches for various neurodegenerative diseases.

\section{Materials and Methods}

\section{Animals}

All animal experiments were approved by the ethics committee for primate research of the National Center of Neurology and Psychiatry in Japan. All experiments were conducted in accordance with institutional guidelines and the National Research Council's Guide for the Care and Use of Laboratory Animals.

\section{Construction of the mutant ataxin 3-120Q vector}

The expansion mutation of CAG repeats within the ataxin 3 gene (more than $\sim 52$ repeats; normal 12-41 repeats) is causative for SCA3/Machado-Joseph disease, which is the most common subtype of autosomal dominant SCAs (Costa and Paulson, 2012). The full-length human ataxin 3 gene with 120 CAGs (ataxin 3-120Q) and a marker gene coding Venus protein were linked by an internal ribosomal entry site (IRES) or a self-cleaving $2 \mathrm{~A}$ peptide (2A) sequence (Fig. 1A). The resultant constructs were inserted into a self-inactivating lentiviral vector carrying the CMV promoter (Riken) using Gateway technol-

Acknowledgments: We thank Drs. Hiroyuki Miyoshi and Atsushi Miyawaki (RIKEN) for providing lentiviral vectors and Venus constructs, Drs. Shinichi Takeda, Takashi Okada, Hironori Okada, Miho Murata, Katsuki Nakamura, Hideyuki Okano (Keio University), and Erika Sasaki (Central Institute for Experimental Animals) for their helpful discussions, and Dr. Toshihide Takeuchi, Dr. Keiko Nakagaki, Shoko Watanabe, Madoka Nakabayashi, Junichi Ueno, Ikuko Takamoto, Kouichi Nakasone, Mami Kumon, Ryoichi Saito, and Akiko Kawanobe for their technical assistance.

Correspondence should be addressed to one of the following: Ikuo Tomioka, Institute for Biomedical Science, Interdisciplinary Cluster for Cutting Edge Research, Shinshu University, 8304 Minami-Minowa, Kami-Ina, Nagano 3994598, Japan. E-mail: tomioka@shinshu-u.ac.jp; or Yoshitaka Nagai, Department of Neurotherapeutics, Osaka University Graduate School of Medicine, 2-2 Yamadaoka, Suita, Osaka 565-0871, Japan. E-mail: nagai@neurother. med.osaka-u.ac.jp; or Kazuhiko Seki, Department of Neurophysiology, National Institute of Neuroscience, National Center of Neurology and Psychiatry, 4-1-1 Ogawahigashimachi, Kodaira, Tokyo 187-8502, Japan. E-mail: seki@ncnp.go.jp. DOI:http://dx.doi.org/10.1523/ENEURO.0250-16.2017

Copyright (C) 2017 Tomioka et al.

This is an open-access article distributed under the terms of the Creative Commons Attribution 4.0 International license, which permits unrestricted use, distribution and reproduction in any medium provided that the original work is properly attributed. ogy (Invitrogen). CAA triplets were inserted every 30 CAG repeats to avoid CAG repeat length mutations.

\section{Lentiviral vector preparation and transduction}

The lentiviral vectors were produced as previously described (Sasaki et al. 2009). Briefly, lentiviruses were generated by cotransfection of lentiviral vectors coding for CMV-ataxin 3-120Q-IRES-Venus or CMV-ataxin 3-120Q2A-Venus, pCAG-HIVgp (Riken), and pCMV-VSV-G (Riken) into 293FT packaging cells (Invitrogen). The medium containing viral particles was spun at $4^{\circ} \mathrm{C}$ and $50,000 \times g$ for $2 \mathrm{~h}$, and the viral pellet was then resuspended in BlastAssist medium (Origio) in $1 / 1000$ of the volume of the original lentiviral vector supernatant. A high titer $\left(1 \times 10^{9}\right.$ to $1 \times$ $10^{11}$ transducing units $/ \mathrm{ml}$ ) of lentiviral vectors was used for the subsequent gene transfer. Four-cell to morulastage embryos were placed in M2 medium (Sigma) supplemented with $0.1 \mathrm{M}$ sucrose, and the viruses were injected into the perivitelline space of embryos using Eppendorf FemtoJet Express (Eppendorf). The embryos were subsequently cultured in BlastAssist medium until embryo transfer.

\section{Embryo collection and transfer}

To determine the ovarian cycle of female marmosets, $100 \mu \mathrm{l}$ of blood was taken from the femoral vein 11 and 13 days after injection of the prostaglandin F2 alpha analog cloprostenol (Cloprostenol C; Fujita Pharmaceuticals), and ovulation days were determined by measuring plasma progesterone concentrations using an enzyme immunoassay (AIA-360; Tosoh Corp.). The day of ovulation ( $\mathrm{d} 0$ ) was designated as the day before the increase in progesterone concentrations from basal levels to $>10$ $\mathrm{ng} / \mathrm{ml}$. The animals were subjected to embryo collection on d 4-7 and embryo transfer on d 2-5. Embryo collection and transfer were performed by nonsurgical methods as previously described (Ishibashi et al. 2013a, b). Briefly, for embryo collection, a blunt tapered 28-G inner stainless steel cannula and a blunt tapered 19-G outer stainless steel cannula were inserted into the uterus with the aid of ultrasonography. While compressing the oviducts, $4 \mathrm{ml}$ of flushing medium was injected through the inner cannula into the uterus. The perfused medium was collected in a dish as it dripped from the outer cannula. For embryo transfer, one or two embryos were transferred to the uterus of each surrogate mother using the same instruments as for embryo collection. After embryo transfer, the recipients were monitored for pregnancy by ultrasound imaging once a month until delivery.

\section{Establishment of ear fibroblast cell lines of transgenic marmosets}

A small piece of tissue from the ear was collected, minced with scissors, and incubated in PBS containing 1 $\mathrm{mg} / \mathrm{ml}$ of collagenase (Wako) for $30 \mathrm{~min}$ at $37^{\circ} \mathrm{C}$. Cells were washed twice by centrifugation and cultured in DMEM containing 10\% fetal bovine serum (Gibco).

\section{DNA/RNA extraction and reverse transcription}

DNA/RNA was isolated from ear fibroblast cells or other tissues of the marmosets using the AllPrep DNA/RNA 
A

CMV-Ataxin3-120Q

-IRES-Venus
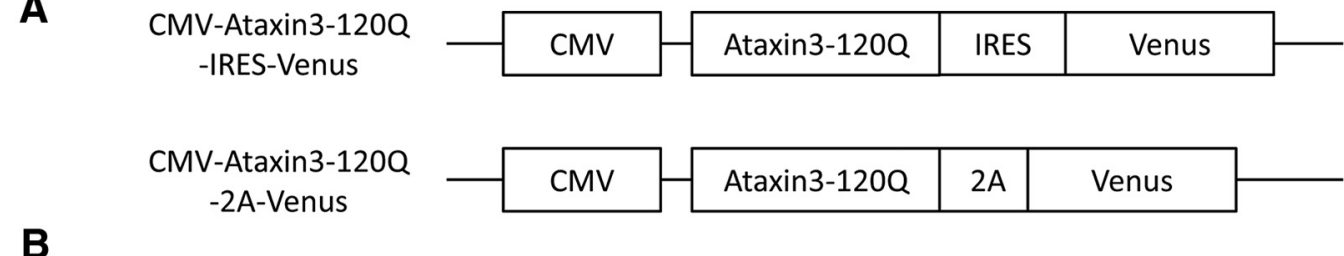

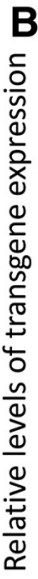

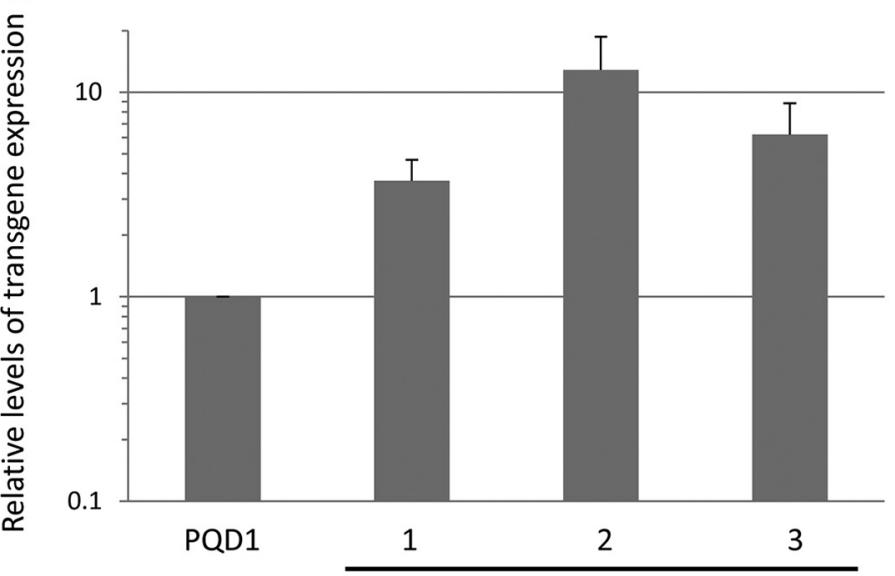

Miscarried fetus

C

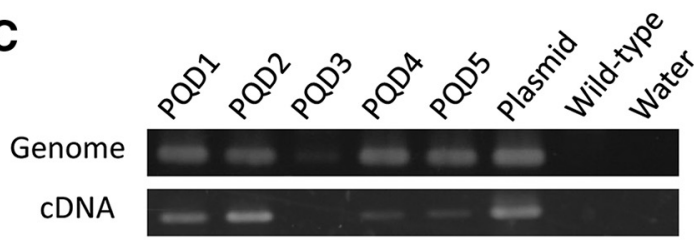

E

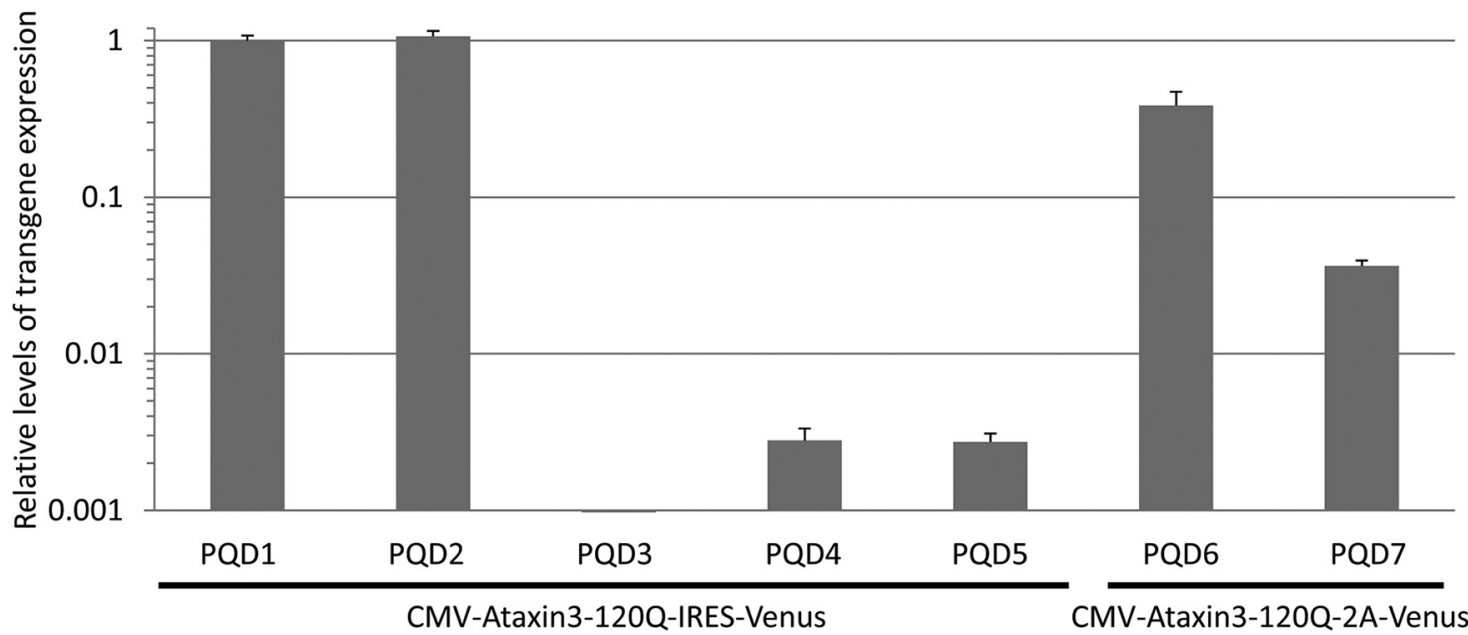

Figure 1. Generation of seven ataxin 3-120Q transgenic marmosets. A, Two types of constructs (CMV-ataxin 3-120Q-IRES-Venus and CMV-ataxin 3-120Q-2A-Venus) were introduced into marmoset embryos. B, Relative levels of transgene expression in PQD1 and three miscarried fetuses are shown. The relative gene expression level in PQD1 was set as 1. C, $\boldsymbol{D}$, Genomic-PCR (top) and RT-PCR (bottom) analyses of each transgenic marmoset transduced with the CMV-ataxin 3-120Q-IRES-Venus construct (C) or the CMV-ataxin 3-120Q-2A-Venus construct $(\boldsymbol{D})$. The results in seven transgenic marmosets (PQD1-7), a positive control (Plasmid), and negative controls (Wild-type and Water) are shown. $\boldsymbol{E}$, Relative levels of transgene expression in ear fibroblast cells of each transgenic marmoset are shown. The relative gene expression level in PQD1 was set as 1.

minikit (Qiagen) according to the manufacturer's instructions. First-strand cDNA was synthesized from RNA using the ReverTra Ace qPCR RT Master Mix with gDNA Remover (Toyobo).
As a negative control, RNA was allowed to react with the cDNA synthesis reaction mixture in the absence of reverse transcriptase. 


\section{PCR analysis}

To detect the mutant ataxin 3 gene, the CMV forward (5'-GTGGATAGCGGTTTGACTCACG-3') and ataxin 3 reverse (5'-TGTTGAGCACAAAGTGAGCCTTC-3') primers were used. To detect the $\beta$-actin gene, the $\beta$-actin forward 1 (5'-AACTGGGACGACATGGAGAAGATC-3') and reverse 2 (5'-GTAGCACAGCTTCTCCTTGATGTC-3') primers were used. Genomic DNA and cDNA were subjected to PCR for 35 cycles of $98^{\circ} \mathrm{C}$ for $10 \mathrm{~s}, 64^{\circ} \mathrm{C}$ for $30 \mathrm{~s}$, and $72^{\circ} \mathrm{C}$ for $30 \mathrm{~s}$ using EmeraldAmp PCR Master Mix (Takara) according to the manufacturer's instructions. For genomicPCR of embryos, which were named PQD (for polyQ diseases), each embryo was collected in $10 \mu$ l of distilled water and boiled at $98^{\circ} \mathrm{C}$ for 5 min. Genomic DNA was subjected to PCR for 35 cycles of $98^{\circ} \mathrm{C}$ for $10 \mathrm{~s}, 64^{\circ} \mathrm{C}$ for $10 \mathrm{~s}$, and $68^{\circ} \mathrm{C}$ for $30 \mathrm{~s}$ using KOD-Plus-Neo (Toyobo) supplemented with $40 \mathrm{ng} / \mu \mathrm{l}$ of T4 gene 32 protein (Wako) at a final concentration according to the manufacturer's instructions.

\section{Real-time PCR analysis}

To quantify the relative expression of transgenes, the Venus forward (5'-TCTTCAAGGACGACGGCAACTAC-3') and reverse (5'-GTTGTGGCTGTTGTAGTTGTACTCC-3') and GAPDH forward (5'-TGACAACAGCCTCAAGATCG-3') and reverse (5'-ACGGTGGTCATGAGTCCTTC-3') primers were used. cDNA was subjected to real-time PCR for 40 cycles of $95^{\circ} \mathrm{C}$ for $5 \mathrm{~s}$ and $60^{\circ} \mathrm{C}$ for $30 \mathrm{~s}$ using SYBR Premix Ex Taq II (Takara) according to the manufacturer's instructions. All real-time PCR results were normalized with GAPDH.

\section{Western blot analysis}

Total protein was extracted from ear fibroblast cells or other tissues from the marmosets using DUALXtract (Dualsystems Biotech). The protein extract was boiled with loading dye before loading onto $10 \%$ polyacrylamide gels (Bio-Rad). After electrophoresis, proteins were transferred onto a PVDF membrane (Bio-Rad) using Trans-Blot (BioRad) followed by blocking in Tris-buffered saline containing $2.5 \%$ bovine serum albumin for $1 \mathrm{~h}$. The membrane was incubated with the mouse monoclonal 1C2 primary antibody (1:3000; Millipore) followed by a peroxidaseconjugated mouse IgG secondary antibody (1:3000; Cell Signaling Technology), and then the proteins were detected with the ECL prime Western Blotting Detection Reagent (GE Healthcare).

\section{Sequencing of CAG repeats}

To detect the presence of CAG repeats within the transgene, the CAG forward 1 (5'-GCTAAGTATGCAAGGTAG TTCCAG- $\left.3^{\prime}\right)$ and reverse 6 (5'-GTCTTCTTCACTCATAGC ATCACC- $3^{\prime}$ ) primers were used. The cDNA was subjected to PCR for 35 cycles of $98^{\circ} \mathrm{C}$ for $10 \mathrm{~s}$ and $68^{\circ} \mathrm{C}$ for $30 \mathrm{~s}$ using Tks Gflex DNA Polymerase (Takara) according to the manufacturer's instructions. Then, the PCR products were sequenced using CAG forward 1, CAG forward 2 (5'-GGAAGAGACGAGAAGCCTAC-3'), CAG reverse 5 (5'-TCCCAAGTGCTCCTGAACTG-3'), and CAG reverse 6 primers to determine the number of CAG repeats. The original plasmids were used as positive controls.

\section{Fluorescent in situ hybridization (FISH) analysis}

FISH analyses were performed according to the previous report (Pinkel et al. 1986) at the Chromocenter (Tottori, Japan). Briefly, ear fibroblast cells of transgenic marmosets were cultured for $4 \mathrm{~h}$ with $0.15 \mu \mathrm{g} / \mathrm{ml}$ of demecolcine (Sigma) and suspended in $0.075 \mathrm{M} \mathrm{KCl}$ for $15 \mathrm{~min}$, followed by fixation in methanol/acetic acid $(3: 1, \mathrm{v} / \mathrm{v})$. The fixed metaphase spreads of the cells were subjected to FISH analysis using biotin-labeled probes for the transgenes, and the bound label was detected using Alexa Fluor 488 streptavidin (Life Technologies). Chromosomal DNA was counterstained with DAPI (Sigma). The images were captured using the Zeiss Axio Imager 2. The FISH signals were analyzed visually by counting $\sim 20-60$ cells per each transgenic marmoset, and chromosomes in which the signal was reproducibly detected in multiple cells were regarded as positive.

\section{Spontaneous motor activity}

The spontaneous motor activities of each marmoset after weaning at 3 months of age were detected and recorded every minute with an infrared sensor (AMN32111, Panasonic). The results were totaled for a day to analyze the amount of spontaneous motor activity and for every hour to analyze diurnal rhythms in the activity.

\section{Bar grip test}

The neuromuscular function of the upper extremities of the marmosets was evaluated using the bar grip test (Smith et al. 1995) after weaning at 3 months of age. The experimenter allowed the marmoset to grasp the bar (bar diameter of $0.7 \mathrm{~mm}$ in a $6 \times 6-\mathrm{mm}$ grid pattern, MK$380 \mathrm{M}$, Muromachi Kikai) with its forelimbs while suspending the marmoset by its tail, and then pulled the tail with a steadily increasing horizontal force. The test was performed three times on each test day, and the maximal grip strength was recorded.

\section{Magnetic resonance imaging}

MRI images of the brain were obtained from six marmosets; PQD1, 3, 4, and 5 at 13.5 months of age, and PQD6 and 7 at 9.5 months of age. The marmoset's head was fixed in an MRI-compatible stereotaxic frame and scanned using a four-channel array coil on a 3-T MRI (Siemens Trio). A three-plane localizer image was obtained to ensure correct positioning of the target images [repetition time (TR), $100 \mathrm{~ms}$; echo time (TE), $5 \mathrm{~ms}$; flip angle (FA), $40^{\circ}$; field of view (FOV), $120 \mathrm{~mm}$; slice thickness, $3 \mathrm{~mm}$ ]. Subsequently, three-dimensional T1weighted images were taken using a magnetization prepared rapid gradient echo sequence (TR, $2300 \mathrm{~ms}$; TE, $2.8 \mathrm{~ms}$; inversion time, $1000 \mathrm{~ms}$; FA, $12^{\circ}$; FOV, $67 \mathrm{~mm}$; image matrix, 192; in-plane voxel size, $\sim 0.3 \mathrm{~mm}$ ).

\section{Fourth ventricle measurement}

T1-weighted MRI images were analyzed using ZioTerm 2009, version 2.0.0.4 (Ziosoft). Using multiplanar reconstruction, tridimensional images were loaded and tilt was corrected manually if necessary to achieve symmetry in the coronal plane. Subsequently, the fourth ventricle was located using the level where the paraflocculus posterior 
Table 1. Production rates of transgenic marmosets

\begin{tabular}{llll}
\hline & CMV-Ataxin3-120Q-IRES-Venus & CMV-Ataxin3-120Q-2A-Venus & Total \\
\hline $\begin{array}{l}\text { Number of embryos transferred } \\
\quad \text { to surrogates }\end{array}$ & 29 & 37 & 66 \\
$\begin{array}{l}\text { Number of surrogates } \\
\text { Number of pregnancies }\end{array}$ & 17 & 23 & 40 \\
$\begin{array}{l}\text { Number of deliveries } \\
\text { Number of births (percentage of }\end{array} \quad 3 \quad$ & 2 & 14 \\
$\quad$ births per embryos transferred) & $5(17.2)$ & $2(5.4)$ & $7(10.6)$ \\
$\quad \begin{array}{l}\text { Number of transgenic animals (percentage } \\
\quad \text { of transgenic animals per birth) }\end{array}$ & $5(100)$ & $2(100)$ & $7(100)$
\end{tabular}

A high titer of lentiviral vector was injected into a total of 66 embryos, followed by embryo transfer to 40 surrogate mothers. A total of 14 surrogates became pregnant, and five surrogates delivered seven offspring.

area was larger as the reference point (stereotaxic reference: interaural $-2.65 \mathrm{~mm}$; Hardman and Ashwell, 2012). After each measurement, a snapshot was taken and all images were compared with confirm the consistency of the selected plane. To standardize the fourth ventricle measurements in relation to the cerebrum, axial MRI T1weighted images were used. The University of Texas Health Science Center at San Antonio Image Tool for Windows software version 3.0 was used to measure the cerebral area of sequential images from the top of the head to the area above the eyeballs of each marmoset. The largest area was selected, and data were expressed as the ratio of the area of the fourth ventricle to that of the cerebrum.

\section{Histochemical analysis of transgenic marmoset tissues}

Postmortem brains, spinal cords, peripheral nerves from the upper and lower limbs, and quadriceps femoris muscle of PQD1 and 2 were fixed in $4 \%$ paraformaldehyde overnight, embedded in paraffin, and sectioned at a thickness of $5 \mu \mathrm{m}$. The sections were stained with hematoxylin and eosin (HE) using standard procedures. Immunohistochemistry was performed with the Ventana XT Discovery (Roche) and the I-View DAB Universal Kit (Roche) according to the manufacturer's instructions. The following antibodies were used: monoclonal anti-1C2 (1: 5000 , Merck Millipore), polyclonal anti-ubiquitin (1:1000, Dako), monoclonal anti-GFAP (1:500, Agilent), and polyclonal anti-calbindin $(1: 16,000$, Swant). Nuclei were stained with hematoxylin. For peripheral nerve analysis, samples were postfixed with $1 \%$ osmium tetroxide, embedded in epoxy resin, sectioned at 1- $\mu \mathrm{m}$ thickness, and stained with toluidine blue. Stained sections were examined under an Eclipse 90i microscope (Nikon).

\section{Histochemical analysis of tibialis anterior muscle}

Skeletal muscle tissue of PQD1 and 2 was processed for pathologic analysis as reported previously (Malicdan et al. 2009). Briefly, serial $10-\mu \mathrm{m}$-thick cryosections were stained with HE and modified Gomori trichrome, for acid phosphatase activity and NADH reductase activity. Stained sections were observed under a BX51 microscope (Olympus), and digitized images were acquired for pathologic analysis using a DP70 digital camera (Olympus).

\section{Biochemical analysis}

Creatine kinase activity in the plasma of PQD2 and four wild-type marmosets was measured using Cicaliquid CK (Kanto Chemical) according to the manufacturer's protocol. The plasma of PQD1 was not successfully obtained at euthanasia.

\section{Production of second-generation transgenic marmoset from PQD1 sperm}

Second-generation transgenic marmoset embryos were produced as described previously (Tomioka et al. 2012; Takahashi et al. 2014). Briefly, PQD1 was induced to ejaculate using a vibrator, and the sperm were collected in TYH medium (Mitsubishi Kagaku latron). Germinal vesicle-stage oocytes of wild-type marmosets were retrieved from follicles (500 $\mu \mathrm{m}$ in diameter) by puncture and cultured for 30 $\mathrm{h}$ in POM (Wako) supplemented with $100 \mathrm{mlU} / \mathrm{mL}$ of follicle-stimulating hormone. Maturated oocytes were fertilized with PQD1 sperm in M2 medium (Sigma-Aldrich) by intracytoplasmic sperm injection using a micromanipulator (Narishige) with the piezo drive system (Primetech). The embryos were subsequently cultured in TYH medium until embryo transfer.

\section{Results}

\section{Production of transgenic marmosets expressing expanded CAG repeats}

Full-length human ataxin 3 cDNA with 120 CAGs (ataxin 3-120Q) was inserted into a self-inactivating lentiviral vector carrying the CMV promoter. We used the lentiviral vector and the CMV promoter to induce efficient integration and high expression of the transgene (Sasaki et al. 2009). Highly efficient transgene integration enables successful transgenesis with a smaller number of embryos, and lentiviral transfection is less invasive than pronucleus injection for DNA delivery to the fertilized eggs or embryos (Chan, 2013). A high titer of lentiviral vector carrying CMVataxin 3-120Q-IRES-Venus or CMV-ataxin 3-120Q-2AVenus (Fig. $1 A$ ) was injected into 29 and 37 embryos, followed by embryo transfer to 17 and 23 surrogate mothers, respectively (Table 1). A total of 14 surrogates became pregnant, and five surrogates delivered a total of seven offspring. These offspring showed no neurologic symptoms at birth. The remaining nine surrogates miscarried midpregnancy after observation of fetal cardiac arrest 
Table 2. Summary of the characteristics of the seven transgenic marmosets

\begin{tabular}{lllll}
\hline ID & Sex & CAG repeats & $\begin{array}{l}\text { Chromosomes with } \\
\text { transgene integration }\end{array}$ & $\begin{array}{c}\text { Disease onset } \\
\text { progression }\end{array}$ \\
\hline PQD1 & Male & 120 & $1,4,17$ & 4 months \\
PQD2 & Female & 120 & $1,17,22$ & 3 months \\
PQD3 & Female & ND & & \\
PQD4 & Female & 120 & & 4 months \\
PQD5 & Female & 120,80 & 7,22 & \\
PQD6 & Female & 120 & & Severe \\
PQD7 & Male & 120,43 & & \\
\hline
\end{tabular}

ND, not determined.

Although all marmosets except PQD3 carried the ataxin 3-120Q transgene, transgenes with other CAG repeat lengths were also found to be expressed in PQD5 and 7, respectively. PQD1, 2, and 6 had several integration sites and cellular mosaicism and developed neurologic symptoms of varying degrees at 3-4 months after birth.

or heartbeat weakness by ultrasound imaging. This high abortion rate indicates that the expression of ataxin $3-120 \mathrm{Q}$ exerts strong cytotoxicity during fetal development, supported by a 3.7- to 12.8-fold higher level of the transgene expression in the miscarried fetuses than that in PQD1 (Fig. 1B). Considering that ear fibroblast cells from all of the surviving offspring showed no visible Venus fluorescence under UV light, only those offspring with lower transgene expression levels appeared to have survived until birth.

\section{Molecular biological analyses of transgenic marmosets}

Transgene integration and expression were examined in ear fibroblast cells from each offspring. All seven offspring carried the ataxin 3-120Q transgene, and six of seven offspring (except PQD3) expressed the transgene (Fig. 1C, D). In particular, three transgenic marmosets, PQD1, 2, and 6, expressed higher levels of the transgene than the other transgenic marmosets, as revealed by quantitative real-time PCR analyses (Fig. 1E). Although all marmosets except PQD3 carried the ataxin 3-120Q transgene, transgenes with other CAG repeat lengths such as 80 and 43 CAG repeats were also found to be integrated in PQD5 and 7, respectively (Table 2). FISH analyses using ear fibroblast cells from PQD1, 2, and 6 showed that each marmoset had several integration sites and cellular mosaicism; positive FISH signals from the transgenes were reproducibly detected in chromosomes 1,4 , and 17 in PQD1; chromosomes 1, 17, and 22 in PQD2; and chromosomes 7 and 22 in PQD6, with patterns that sometimes varied among different cells (Table 2).
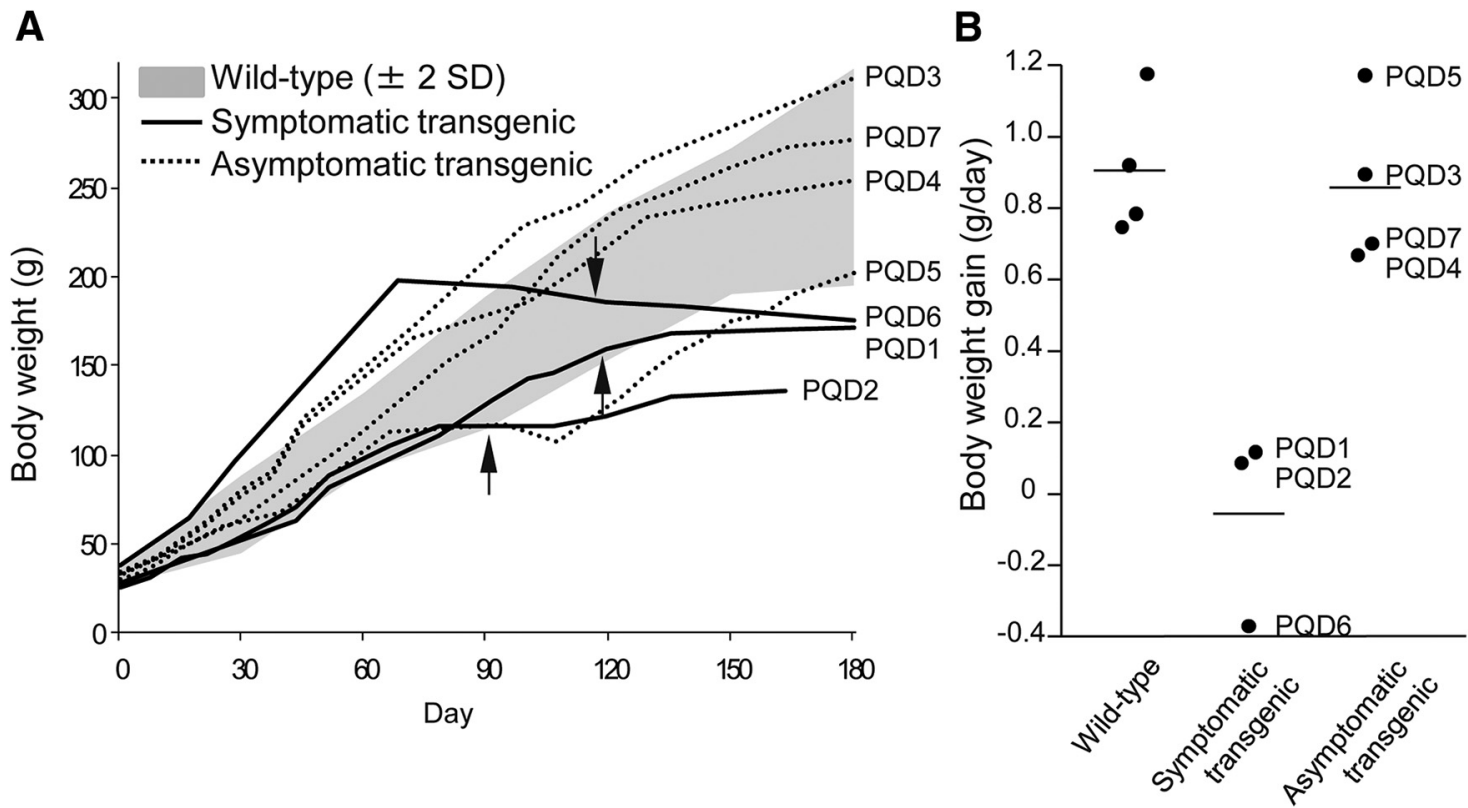

Figure 2. Age-dependent changes in body weight and body weight gain. A, Age-dependent changes in the body weight of wild-type marmosets, symptomatic transgenic marmosets (PQD1, 2, and 6), and asymptomatic transgenic marmosets (PQD3, 4, 5, and 7). Arrows indicate the onset of neurologic symptoms of the three symptomatic transgenic marmosets. Averages \pm 2 SD of body weight in wild-type marmosets $(n=4)$ are indicated as gray areas. $\boldsymbol{B}$, Body weight gain of wild-type marmosets, symptomatic transgenic marmosets, and asymptomatic transgenic marmosets at 5-6 months of age. Each symbol represents an individual marmoset. Horizontal bars indicate the group average. 

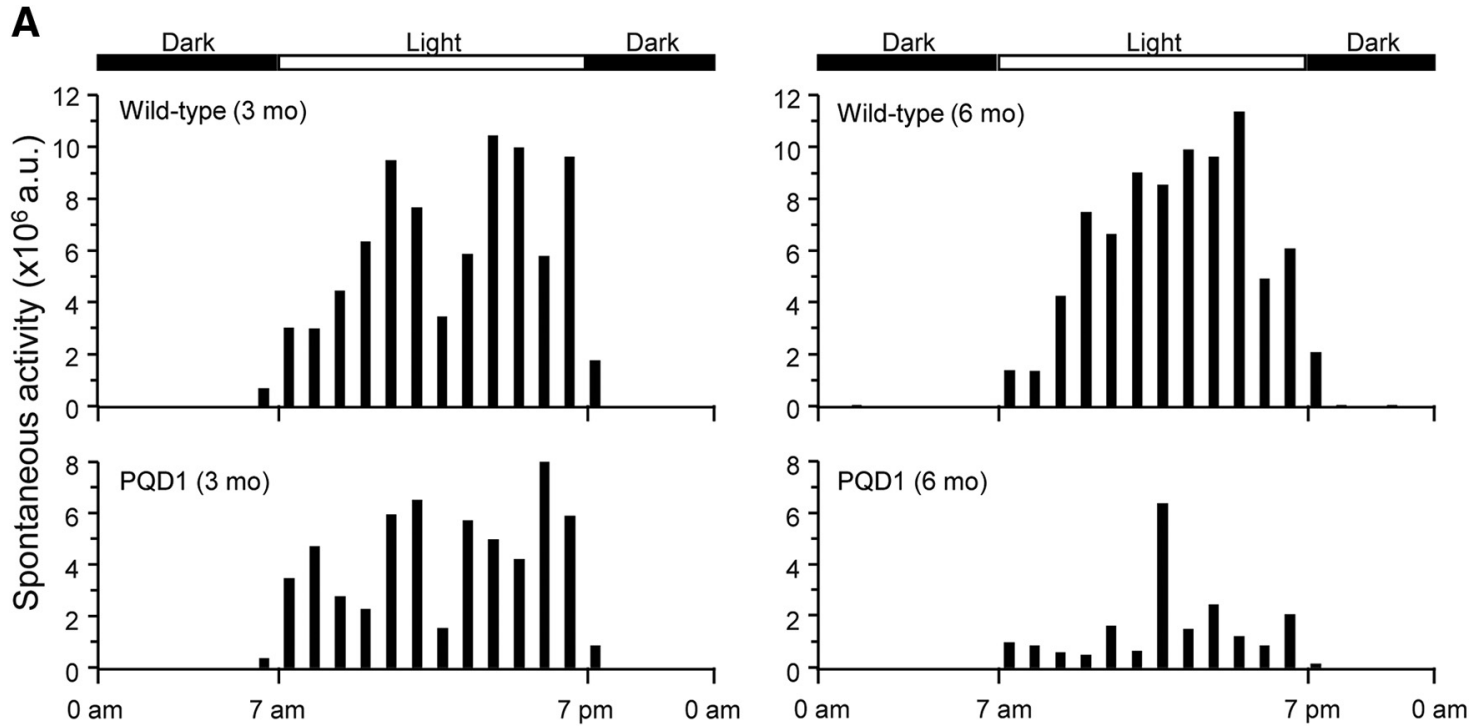

B
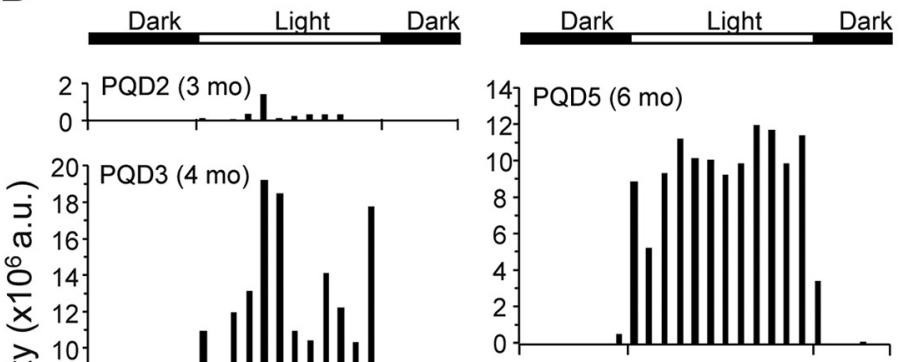

C
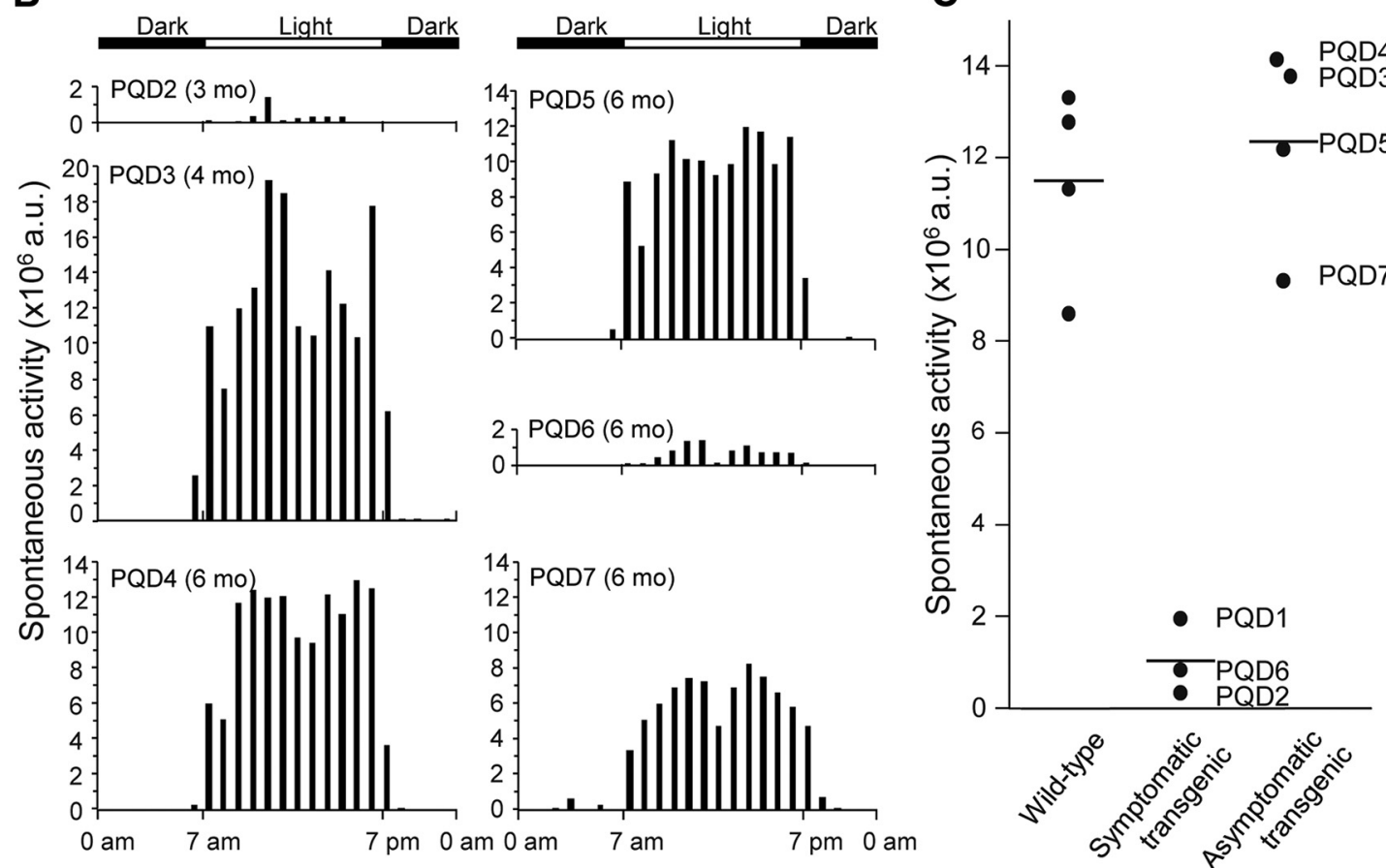

Figure 3. Transgenic marmosets show progressive neurologic symptoms. A, Age-dependent changes in 24-h spontaneous activity of a wild-type marmoset and PQD1 transgenic marmoset at 3 and 6 months. $B, 24-h$ spontaneous activity of PQD2 at 3 months, PQD3 at 4 months, and PQD4-7 at 6 months of age. C, 24-h spontaneous activity levels of PQD1 at 5-6 months of age, PQD2 at 3 months, and PQD6 at 5-6 months of age, compared with those of wild-type marmosets and asymptomatic transgenic marmosets at 5-6 months of age. Each symbol represents an individual marmoset. Horizontal bars indicate the group average.

\section{Behavioral analyses of transgenic marmosets}

Although all animals showed no symptoms at birth and grew normally, PQD1, 2, and 6, which expressed ataxin 3-120Q at higher levels, developed neurologic symptoms of varying degrees at 3-4 months after birth (Table 2). In the early stages of the disease, these three transgenic marmosets often slipped down from the grids of the cage, and their jumping ability was slightly but noticeably decreased, and the symptoms progressed to a severe movement disability accompanied by contractures of the legs and arms in the later stages. These symptomatic transgenic marmosets showed gradual decreases in body weight gain and almost no further weight gain beyond 3-4 months of age (Fig. 2A, B). The levels of body weight gain were significantly lower than those of wild-type marmosets and asymptomatic transgenic marmosets at 5-6 months of age (Fig. $2 B ; p<0.01$ ). Spontaneous activity monitoring revealed a progressive decline in activity in PQD1 compared with a wild-type marmoset, without apparent diurnal rhythm alterations (Fig. $3 A$ ). Spontaneous 


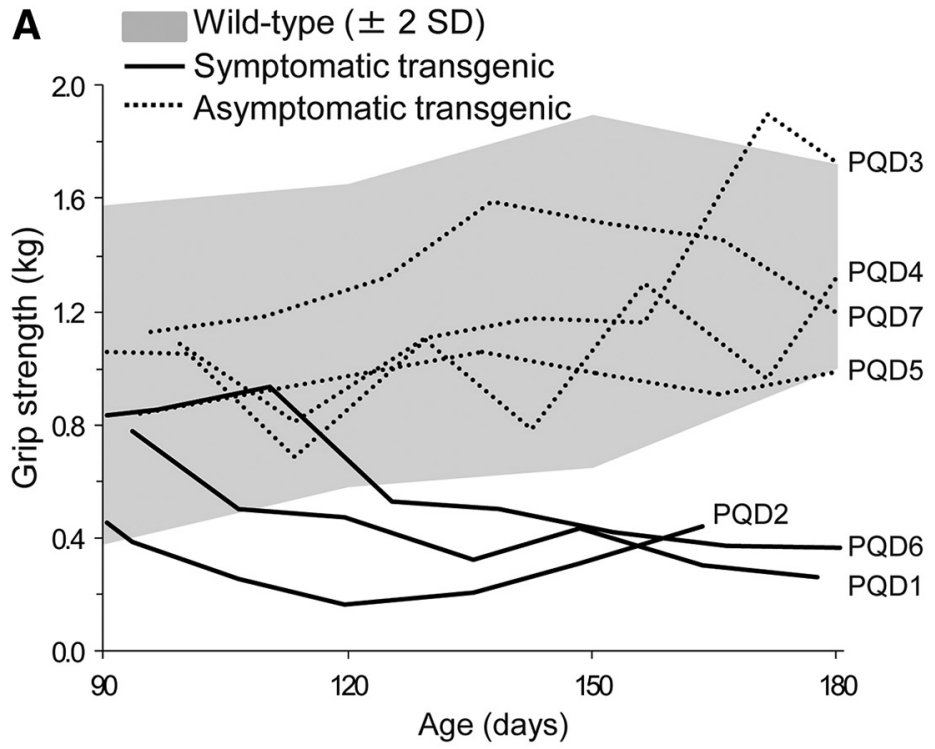

B

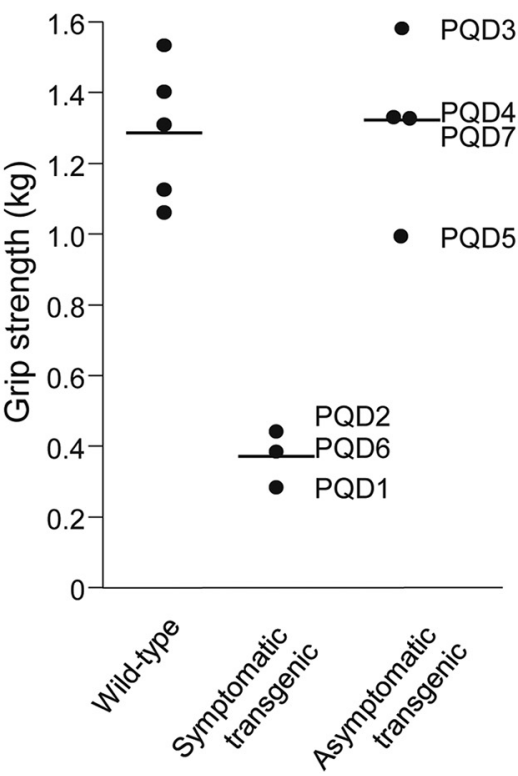

Figure 4. Age-dependent changes in grip strength. $\boldsymbol{A}$, Age-dependent changes in grip strength of wild-type marmosets, symptomatic transgenic marmosets (PQD1, 2, and 6), and asymptomatic transgenic marmosets (PQD3, 4, 5, and 7). Averages \pm 2 SD of grip strength in wild-type marmosets $(n=4)$ are indicated as gray areas. $\boldsymbol{B}$, Grip strength of wild-type marmosets, symptomatic transgenic marmosets, and asymptomatic transgenic marmosets at 5-6 months of age. Each symbol represents an individual marmoset. Horizontal bars indicate the group average.

activity levels of PQD2 and 6 after onset of symptoms were also significantly lower than those of wild-type marmosets and asymptomatic transgenic marmosets (Fig. $3 B, C ; p<0.01)$. Grip strengths of PQD1, 2, and 6 gradually decreased (Fig. $4 A$ ) and were significantly lower than those of wild-type marmosets at 5-6 months of age (Fig. $4 B ; p<0.01$ ). As shown in these figures, all behavioral measurements as well as the body weight of asymptomatic transgenic marmosets were comparable with that of wild-type marmosets. For example, the body weight of asymptomatic transgenic marmosets showed a progressive increase throughout the whole period of measurement (Fig. 2A), and the level of body weight gain was not different from that of the wild-type (Fig. 2B). Similarly, no significant difference was found in the spontaneous activity (Fig. $3 C$ ) or grip strength (Fig. $4 B$ ).

\section{Molecular biological analyses of symptomatic transgenic marmosets}

Because of their severe difficulty in eating by themselves and their progressive body weight loss, PQD1 and PQD2 were euthanized at 13.5 and 5.5 months of age, respectively, and were subjected to biochemical and pathologic analyses. Integration and expression of the mutant ataxin 3 transgene in the major organs of PQD1 and 2 were examined. Genomic and RT-PCR analyses confirmed integration and expression of the transgene in all analyzed organs of both PQD1 and 2 (Fig. 5A). Western blot analyses revealed expression of the expanded polyQ protein in the heart, kidney, lung, spleen, skeletal muscle, and various brain regions in PQD1 and the heart, liver, skeletal muscle, and various brain regions in PQD2 (Fig. 5B).

\section{Pathologic analyses of symptomatic transgenic marmosets}

Noninvasive MRI analyses were performed on two of the three symptomatic marmosets, PQD1 at 13.5 months of age and PQD6 at 9.5 months of age, and four asymptomatic transgenic marmosets at the ages corresponding to either PQD1 or 6 . MRI scanning revealed significant enlargement of the fourth ventricle in one of the symptomatic marmosets, PQD1, compared with asymptomatic transgenic marmosets, suggesting cerebellar atrophy (Fig. 6A-C; relative fourth ventricle area: PQD1, 0.348 arbitrary units (au); PQD6, 0.169 au; average of four asymptomatic transgenic marmosets, $0.235 \pm 0.049 \mathrm{au}$ ). Consistent with the MRI finding, immunohistochemical analysis of PQD1, which was euthanized immediately after the MRI scanning, revealed a marked loss of Purkinje cells (Fig. $6 D, E$ ) accompanied by gliosis (Fig. $6 F, G$ ) in the cerebellum. In addition, degeneration of lower motor neurons and eosinophilic intranuclear inclusions were evident in the anterior horn of the cervical and lumbar spinal cord of PQD1 (Fig. 6H). Importantly, these inclusions were immunoreactive for the 1C2 antibody (Fig. 6/, J), which specifically recognizes the expanded polyQ stretch, and for the ubiquitin antibody (Fig. 6K). Mild gliosis was also detected in the spinal cord of PQD1 (Fig. 6L, M). The cerebrum and brainstem did not show significant pathology (Fig. $6 \mathrm{~N}, \mathrm{O}$ ) except for scarce 1C2-positive inclusions observed in the brainstem (Fig. 6P).

On the contrary, the immunohistochemical analysis of PQD2, on which MRI analysis was not performed, did not show significant cerebellar pathology (Fig. 7A, B). However, intranuclear eosinophilic and 1C2-positive inclu- 
A
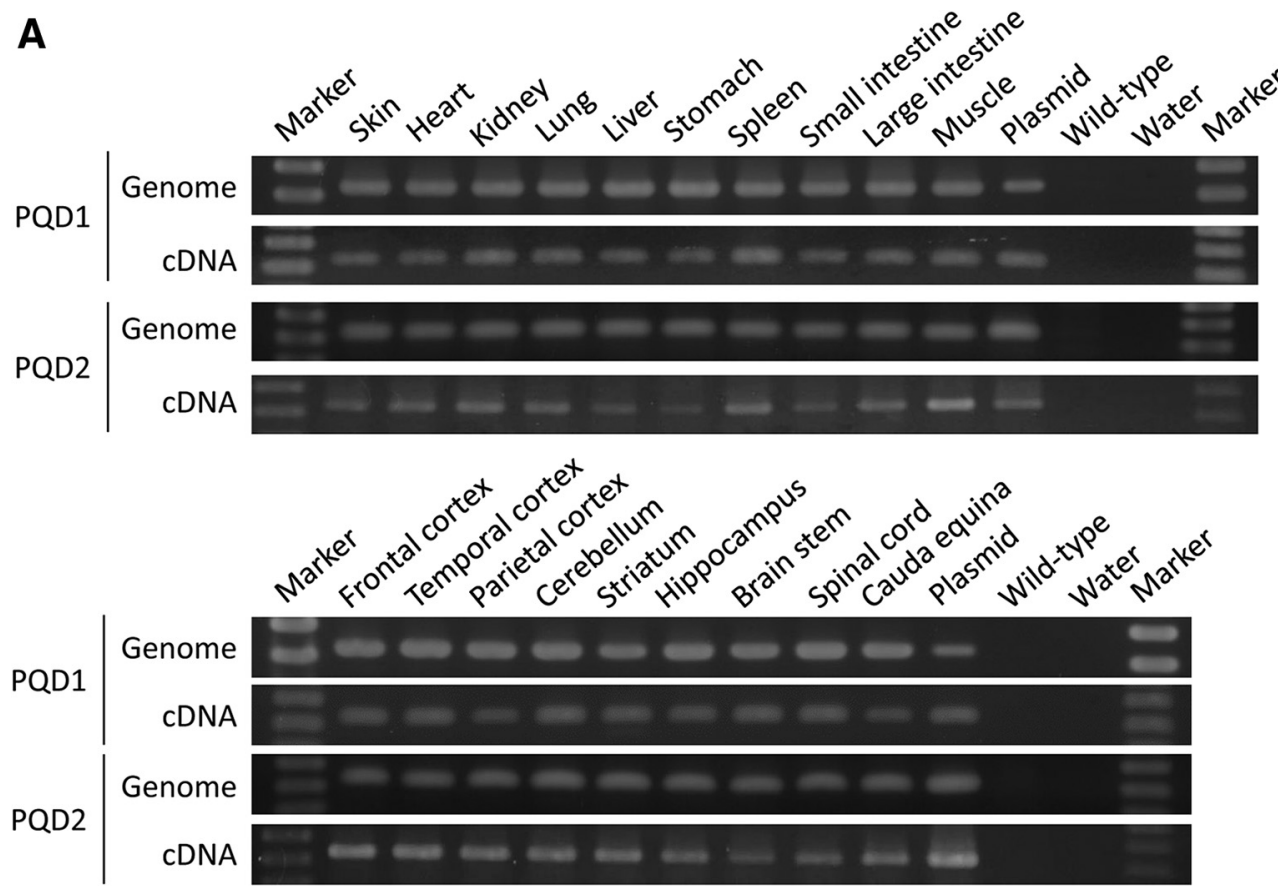

B

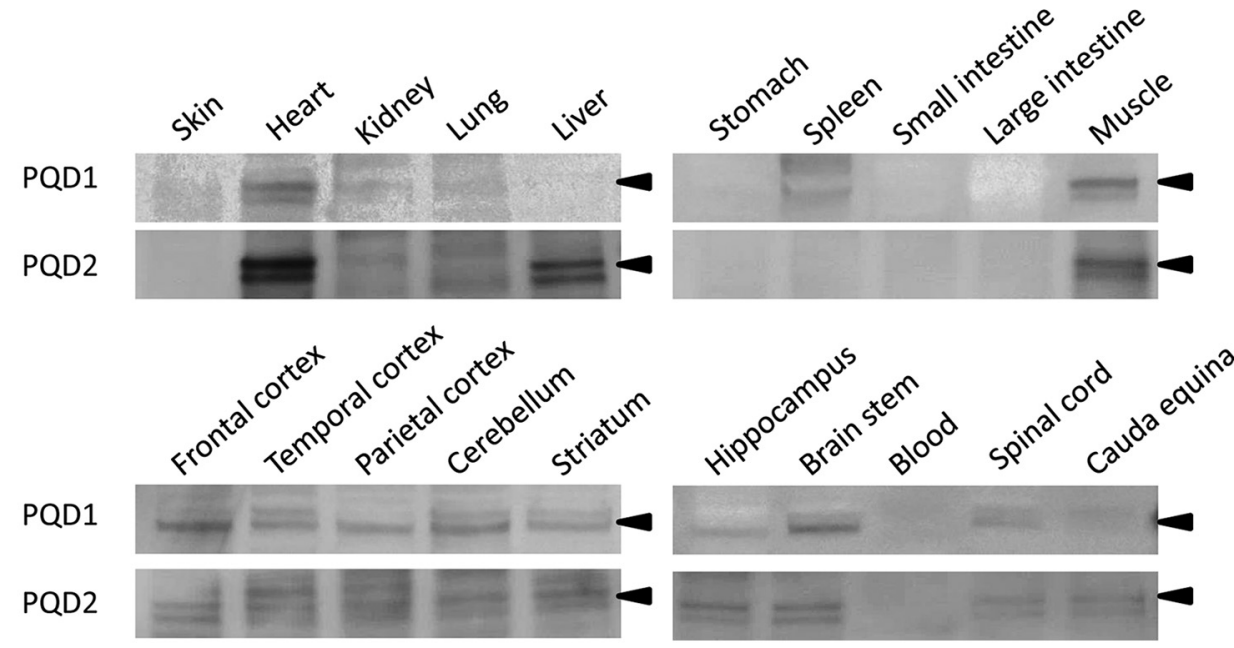

Figure 5. Expression of ataxin 3-120Q in transgenic marmosets PQD1 and 2. $\boldsymbol{A}, \boldsymbol{B}$, Genomic-PCR (top) and RT-PCR (bottom) analyses $(\boldsymbol{A})$ and Western blot analysis $(\boldsymbol{B})$ of the major organs and various brain regions of PQD1 and 2 . A positive control (Plasmid) and negative controls (Wild-type and Water) were also included. Arrowheads indicate the position of the ataxin 3-120Q band.

sions similar to those observed in PQD1 were frequently observed in the brainstem (Fig. 7C, D) and spinal cord (Fig. 7F, G) of PQD2. These inclusions were also positive for the ubiquitin antibody (Fig. 7E, H). The spinal cord also showed neurodegeneration (Fig. 7F) accompanied by mild gliosis (Fig. 7I). Although the cerebrum of PQD2 did not show significant pathology in HE staining (Fig. 7J), ubiquitin-positive but 1C2-negative inclusions were observed (Fig.7K).

These results suggest that the symptomatic transgenic marmosets PQD1 and 2 successfully recapitulated the common pathologic characteristics of the polyQ disease patients, namely, neurodegeneration and the formation of intranuclear inclusions composed of abnormal protein with polyQ expansion (Table 3).

Our transgenic marmosets also exhibited several additional pathologies in the peripheral nerves and muscles, probably because of widespread expression of the expanded polyQ protein under the CMV promoter. Acute axonal degeneration was observed in the peripheral nerves of the upper and lower limbs of PQD1 and 2 (Fig. $8 A, B)$. PQD1 and 2 also exhibited muscular pathology consistent with chronic myopathic changes caused by the ataxin 3 protein with expanded polyQ stretch (Fig. $8 \mathrm{C}-F$ and Table 3). Degenerating myofibers with eosinophilic intranuclear inclusions were observed in PQD2 (Fig. 8C). 

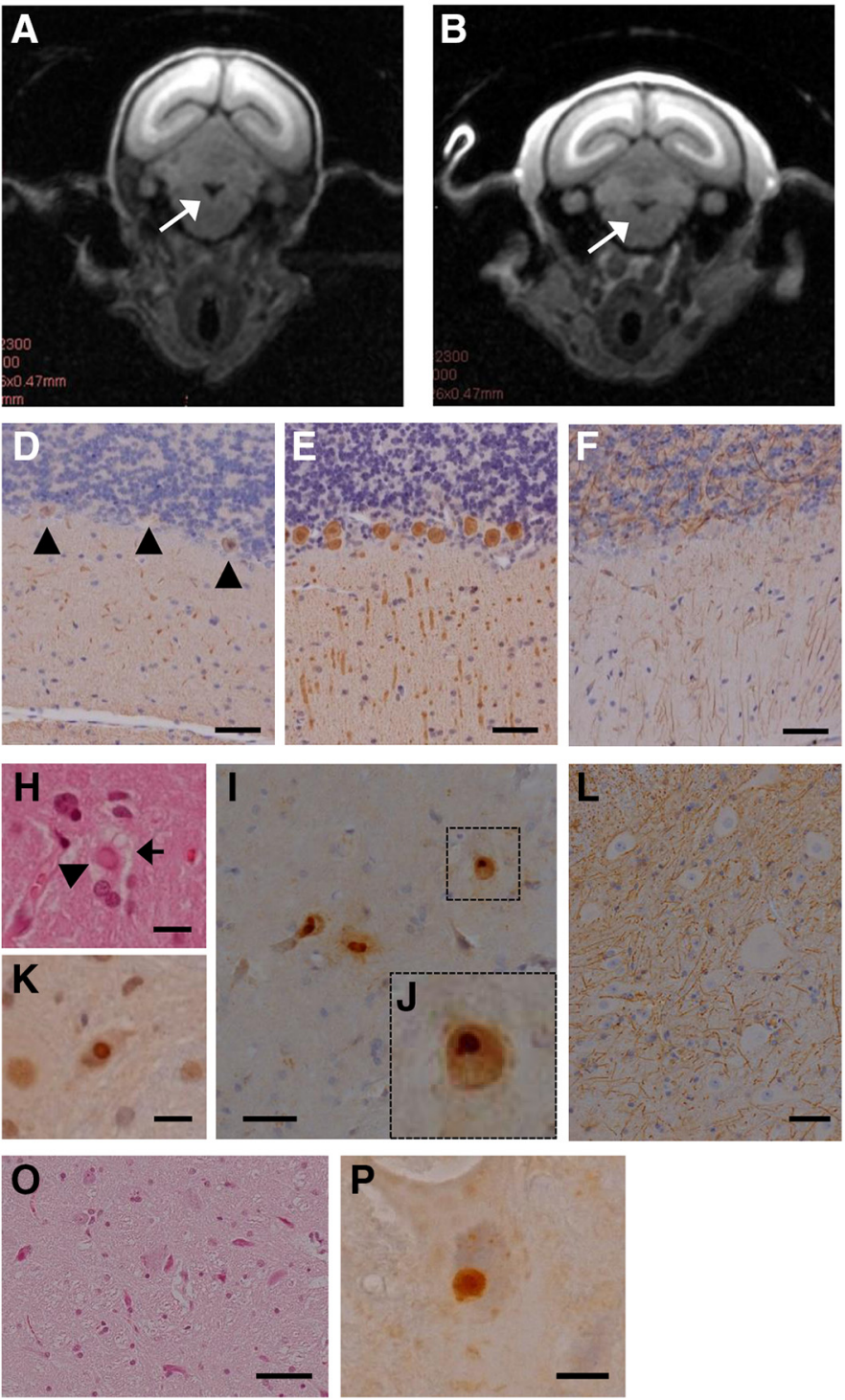

Figure 6. Cerebellar atrophy and neurodegeneration accompanied by polyQ protein inclusions in the brains of symptomatic transgenic marmoset PQD1. $\boldsymbol{A}, \boldsymbol{B}$, Coronal MRI sections of the brain of PQD1 $(\boldsymbol{A})$ and asymptomatic marmoset PQD5 $(\boldsymbol{B})$. The arrow indicates the fourth ventricle. $\boldsymbol{C}$, Quantitative analyses of brain MRIs of transgenic marmosets. The bar indicates the mean of PQD3-5 and 7. $\boldsymbol{D}, \boldsymbol{E}$, Anti-calbindin staining of the cerebellum of PQD1 $(\boldsymbol{D})$ or a control wild-type marmoset $(\boldsymbol{E})$. Arrowheads indicate degenerated Purkinje cells. $\boldsymbol{F}, \boldsymbol{G}$, Anti-GFAP staining of the cerebellum of PQD1 (F) or a control wild-type marmoset $(\boldsymbol{G})$. $\boldsymbol{H}-\boldsymbol{K}$, Hematoxylin and eosin ( $\mathrm{HE} ; \boldsymbol{H})$, anti-1C2 $(\boldsymbol{I}$ and $\boldsymbol{J})$, and anti-ubiquitin $(\boldsymbol{K})$ staining of the spinal cord of PQD1. In $\boldsymbol{H}$, the arrow indicates a degenerated anterior horn cell, and the arrowhead indicates an inclusion body. A magnified image of a 1C2-positive inclusion $(\boldsymbol{I})$ is shown in $\boldsymbol{J}$. $\boldsymbol{L}, \boldsymbol{M}$, Anti-GFAP staining of the spinal cord of PQD1 $(\boldsymbol{L})$ and a control wild-type marmoset $(\boldsymbol{M})$. $\boldsymbol{N}$, HE staining of the cerebrum of PQD1. $\mathbf{O}, \boldsymbol{P}, \mathrm{HE}(\mathbf{O})$ and anti-1C2 $(\boldsymbol{P})$ staining of the brainstem of PQD1. Scale bars: $100 \mu \mathrm{m}$ in $\boldsymbol{D}-\boldsymbol{H}$ and $\boldsymbol{K} ; 200 \mu \mathrm{m}$ in $\mathbf{G} ; 50 \mu \mathrm{m}$ in $\boldsymbol{I}, \boldsymbol{L}, \boldsymbol{M}$, and $\mathbf{O} ; 250 \mu \mathrm{m}$ in $\mathrm{N}$; and $10 \mu \mathrm{m}$ in $\boldsymbol{P}$.

The muscles of PQD1 exhibited severe degeneration accompanied by marked fat deposition, and thus it was difficult to detect these changes, including eosinophilic inclusions using cryosections of the tibialis anterior muscle. Immunohistochemical analysis of the PFA-fixed sections with the $1 \mathrm{C} 2$ antibody, however, demonstrated abundant accumulation of the inclusions with expanded polyQ protein in both PQD1 and 2 in the quadriceps femoris muscles (Fig. 8G, H). These inclusions were also positive for the ubiquitin antibody (Fig. 8/, J). The creatine kinase level of PQD2 was mildly elevated (226 IU/I) com- pared with those of wild-type marmosets $(88.2 \pm 83.6$ $\mathrm{IU} / \mathrm{I}, n=4)$.

\section{Germline transmission of the transgene}

To obtain second-generation offspring of the transgenic marmosets, the ejaculated sperm of PQD1 was collected after sexual maturity at the age of 11 months and injected into the oocytes of a wild-type marmoset by intracytoplasmic sperm injection. A total of 40 embryos were transferred to 17 surrogate mothers. As a result, four offspring (named PQD1-1, 1-2, 1-3, and 1-4) were ob- 

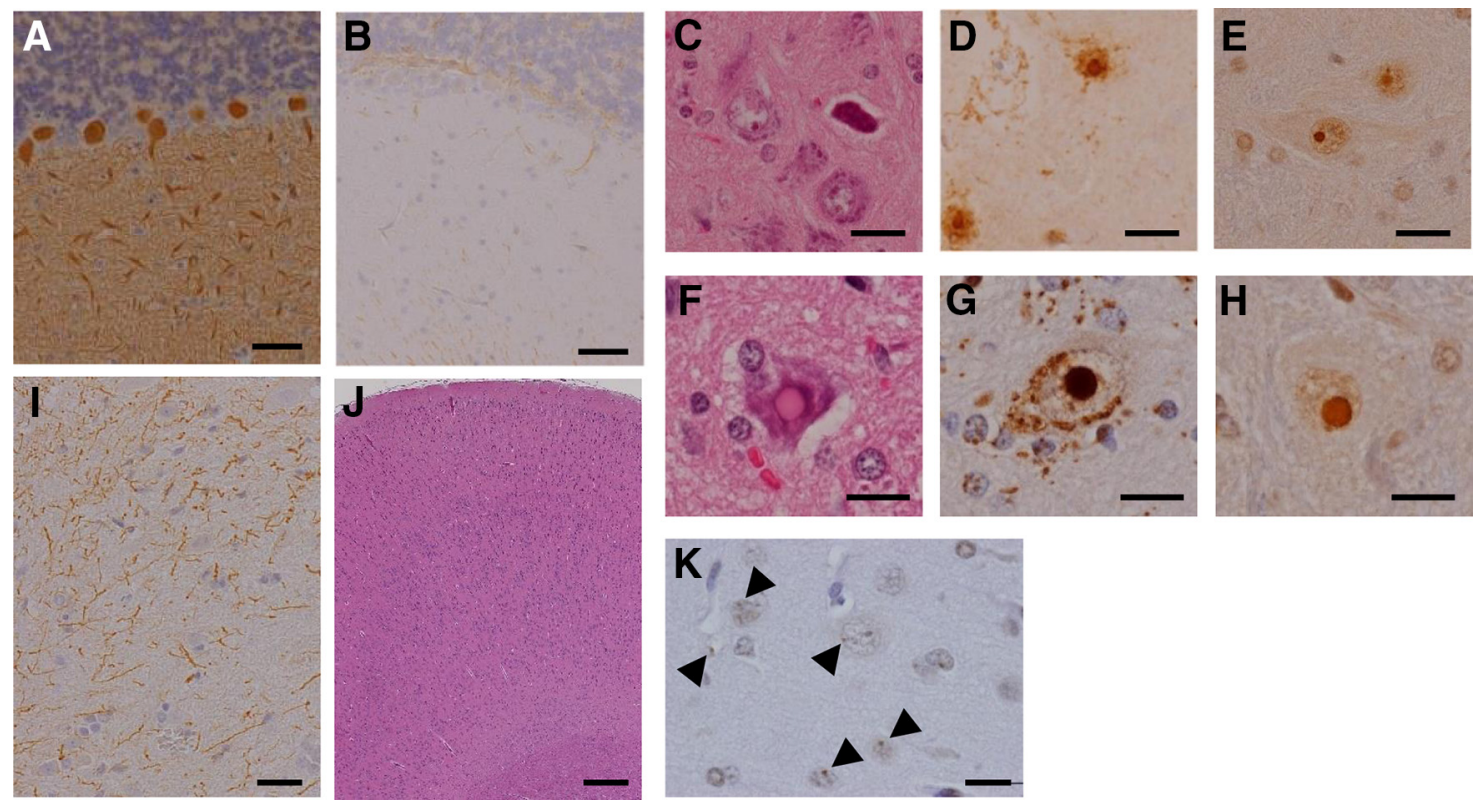

Figure 7. Neurodegeneration and polyQ protein inclusions in the brain and spinal cord of symptomatic transgenic marmoset PQD2. $\boldsymbol{A}, \boldsymbol{B}$, Anti-calbindin $(\boldsymbol{A})$ and GFAP $(\boldsymbol{B})$ staining of the cerebellum of PQD2. $\boldsymbol{C}-\boldsymbol{H}, \mathrm{HE}(\boldsymbol{C}$ and $\boldsymbol{F})$, anti-1C2 (D and $\boldsymbol{G})$, and anti-ubiquitin $(\boldsymbol{E}$ and $\boldsymbol{H})$ staining of the brainstem $(\boldsymbol{C}-\boldsymbol{E})$ and spinal cord $(\boldsymbol{F}-\boldsymbol{H})$ of PQD2. I, Anti-GFAP staining of the spinal cord of PQD2. $\boldsymbol{J}, \boldsymbol{K}, \mathrm{HE}$ $(\boldsymbol{J})$ and anti-ubiquitin $(\boldsymbol{K})$ staining of the cerebrum of PQD2. These inclusions (arrowheads in $\boldsymbol{K}$ ) were not immunoreactive for the $1 \mathrm{C2}$ antibody. Scale bars: $100 \mu \mathrm{m}$ in $\boldsymbol{A}, \boldsymbol{B}$, and $\boldsymbol{F}-\boldsymbol{H} ; 5 \mu \mathrm{m}$ in $\boldsymbol{C}-\boldsymbol{E} ; 50 \mu \mathrm{m}$ in $\boldsymbol{I} ; 250 \mu \mathrm{m}$ in $\boldsymbol{J}$; and $10 \mu \mathrm{m}$ in $\boldsymbol{K}$.

tained from two surrogate mothers. Transgene integration and expression were examined in ear fibroblast cells from each offspring. All offspring carried and expressed the ataxin 3-120Q transgene with 120 CAG repeats (Fig. 8). Moreover, higher or similar levels of transgene expression in PQD1 were confirmed in PQD1-1 and 1-3, as revealed by quantitative real-time PCR analyses (Fig. 9B). FISH analyses using ear fibroblast cells showed that transgenes were integrated into chromosomes $1,3,10$, and 16 in PQD1-1; chromosomes 4 and 16 in PQD1-2; and chromosomes 3 and 22 in PQD1-3 and 1-4. These results suggested that the transgene inserted chromosomes were different between PQD1 and each second-generation offspring, which is considered to be caused by mosaicism in PQD1.

Table 3. Summary of the pathologic findings of PQD1 and 2

\begin{tabular}{|c|c|c|c|c|}
\hline & \multicolumn{2}{|c|}{ PQD1 } & \multicolumn{2}{|c|}{ PQD2 } \\
\hline & \multicolumn{2}{|c|}{13.5 months } & \multicolumn{2}{|c|}{5.5 months } \\
\hline & Degeneration & Inclusion & Degeneration & Inclusion \\
\hline Cerebrum & - & - & - & + \\
\hline Cerebellum & ++ & - & - & - \\
\hline Brain stem & - & \pm & - & + \\
\hline Spinal cord & ++ & ++ & ++ & ++ \\
\hline $\begin{array}{c}\text { Peripheral } \\
\text { nerves }\end{array}$ & ++ & N/E & ++ & N/E \\
\hline $\begin{array}{l}\text { Skeletal } \\
\text { muscles }\end{array}$ & +++ & ++ & +++ & ++ \\
\hline
\end{tabular}

Two marmosets among the three symptomatic transgenic marmosets were subjected to immunohistochemical analysis. Note that cerebellar degeneration was observed in PQD1. N/E: not examined.

\section{Discussion}

A significant milestone in modeling human disease in nonhuman primates using the transgenic technique is the earlier report of transgenic HD rhesus monkey models that express the polyQ-expanded huntingtin gene (Yang et al. 2008). Although these transgenic monkeys showed some polyQ-disease-like features, including intranuclear inclusions in the brain, their symptoms appeared immediately after birth, and unfortunately, the symptomatic animals died without germline transmission of the transgenes. In a more recent study, the same group has succeeded in producing transgenic HD rhesus monkey models exhibiting slower onset and progression with the onset of behavioral phenotypes at 8 or 16 months of age (Chan et al. 2014, 2015). Furthermore, they have confirmed germline transmission of the transgene in the second-generation offspring generated from one of the founder monkeys. Unfortunately, however, the number of CAG repeats in the transgene of one of the two offspring obtained in the study was shortened to 53-61 repeats compared with $72-74$ repeats in the founder monkey and the other offspring, likely caused by the instability of the triplet repeat sequence (Moran et al. 2015). Although the transgene was expressed under the control of the human $H T T$ promoter in this founder monkey and thus is expected to recapitulate the late onset and gradual progression of adult HD, establishing symptomatic HD monkey model colonies in a time-efficient manner for future studies from this founder monkey could be challenging.

In the present study, we successfully generated a transgenic marmoset model of the polyQ diseases with no symptoms at birth, which exhibited progressive motor impairment within several months after the disease onset 

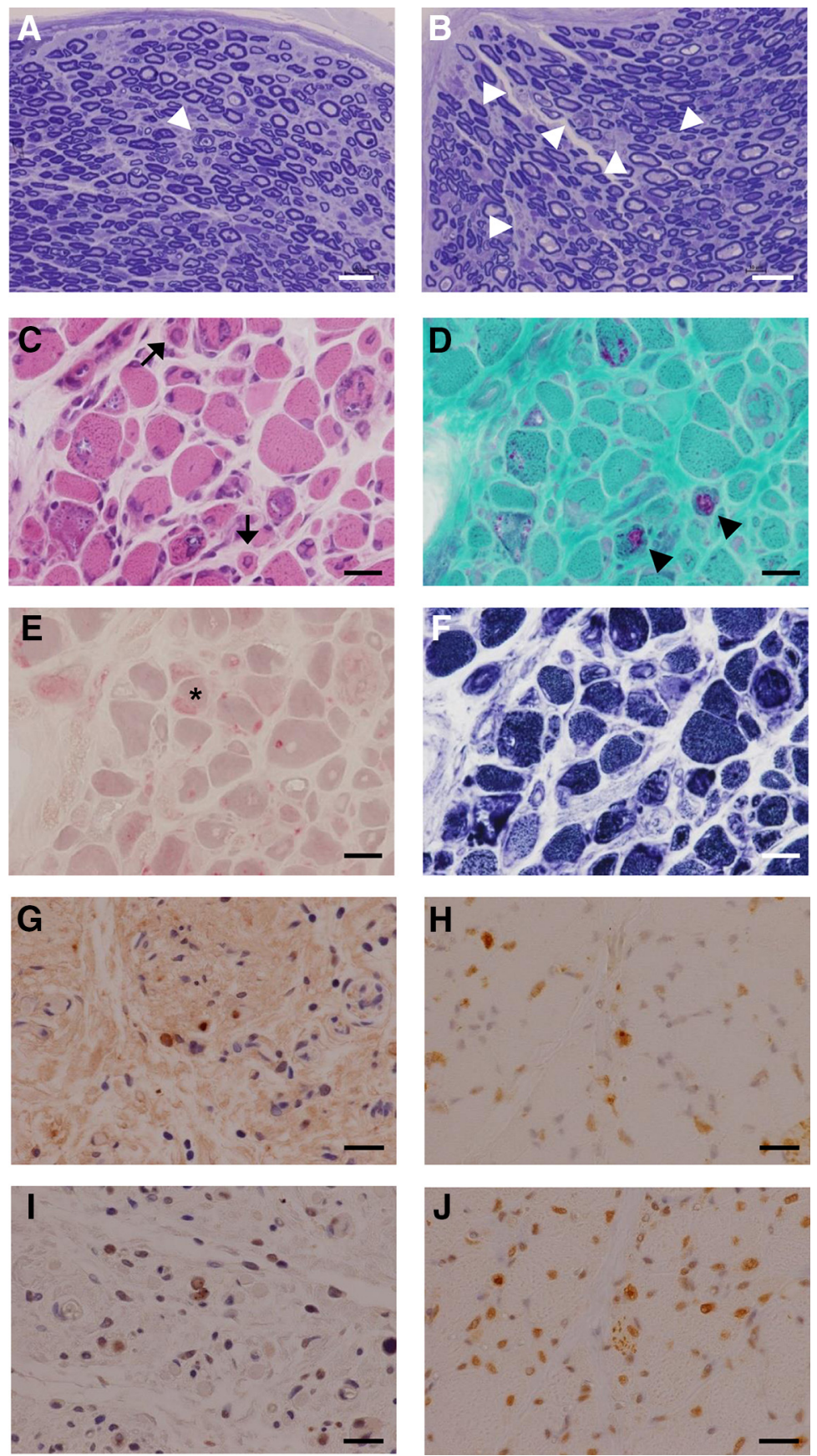

Figure 8. Pathology of the peripheral nerves and muscles of PQD1 and 2. $\boldsymbol{A}, \boldsymbol{B}$, Toluidine blue staining of the peripheral nerves of the upper limbs of PQD1 $(\boldsymbol{A})$ and PQD2 $(\boldsymbol{B})$. Arrowheads indicate axonal degeneration. $\boldsymbol{C}-\boldsymbol{F}, \mathrm{HE}(\boldsymbol{C})$, modified Gomori trichrome $(\boldsymbol{D})$, acid phosphatase activity $(\boldsymbol{E})$, and NADH reductase activity $(\boldsymbol{F})$ staining of the tibialis anterior muscle of PQD2. Marked fiber size variation and endomysial fibrosis were observed. Fibers with centrally placed large nuclei and intranuclear inclusions (arrows in $\boldsymbol{C}$ ) or rimmed vacuoles (arrowheads in $\boldsymbol{D}$ ) and acid phosphatase-positive fibers (asterisk in $\boldsymbol{E}$ ) were also observed. The intermyofibrillar network was disorganized in many fibers $(\boldsymbol{F})$. Dystrophic changes, group atrophy, and fiber-type grouping were not observed. $\mathbf{G}-\boldsymbol{J}$, Anti-1C2 (G and $\boldsymbol{I})$ and anti-ubiquitin $(\boldsymbol{H}$ and $\boldsymbol{J})$ staining of the quadriceps femoris muscle of PQD1 ( $\boldsymbol{G}$ and $\boldsymbol{H})$ and PQD2 (I and $\boldsymbol{J})$. Scale bars: $100 \mu \mathrm{m}$ in $\boldsymbol{A}$ and $\boldsymbol{B} ; 20 \mu \mathrm{m}$ in $\boldsymbol{C}-\boldsymbol{J}$.

at 3-4 months of age. To produce transgenic marmoset models that are expected to develop neurologic symptoms at an early age, we decided to use ataxin 3 with a hyperexpanded $120 \mathrm{CAG}$ repeat, as well as the lentiviral vector carrying CMV promoter, which is the only method established so far and currently accepted to generate transgenic marmosets (Sasaki et al. 2009). Hyperexpansion of the CAG repeat above the human disease range (52-86 repeats) has been widely applied to reproduce neurodegenerative diseases in short-lived rodent models, because the number of CAG repeats is inversely correlated with disease onset in the polyQ diseases (Davies 

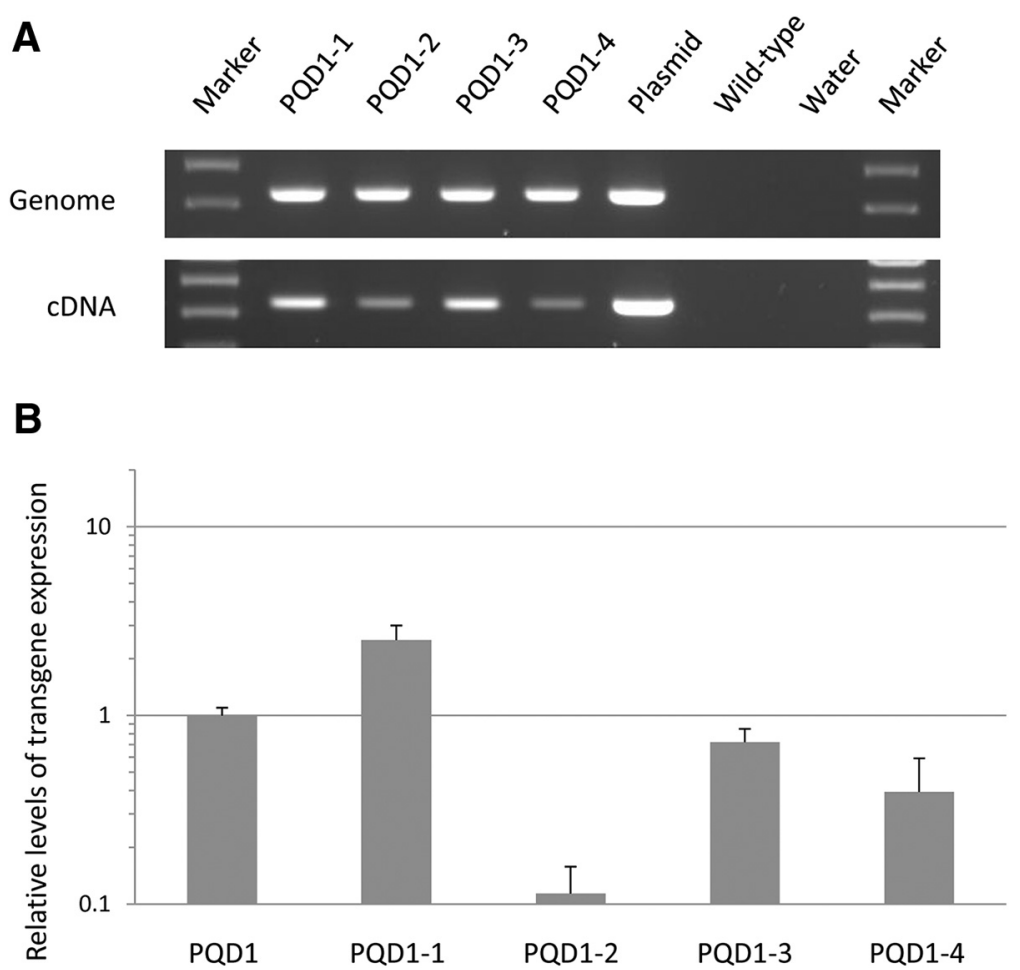

Figure 9. Germline transmission of the ataxin 3-120Q transgenes. A, Genomic-PCR (top) and RT-PCR (bottom) analyses in second-generation transgenic offspring. The results in PQD1-1, 1-2, 1-3, and 1-4, a positive control (Plasmid), and negative controls (Wild-type and Water) are shown. B, Relative levels of transgene expression in ear fibroblast cells of each second-generation transgenic offspring are shown. The relative gene expression level in PQD1 was set as 1.

et al. 1997; Watase et al. 2002; Boy et al. 2010). In addition, because triplet repeat sequences are known to show instability during DNA replication (Lenzmeier and Freudenreich, 2003), CAA triplets that also encode glutamine were introduced every 30 CAG repeats to avoid the mutation. As a result, all the transgenic marmosets analyzed in our study were confirmed to carry a transgene with the original length of 120 CAG repeats, although some variations in the CAG repeat length were found in PQD5 and 7 (Fig. 1D). Through these technical approaches, we successfully generated transgenic marmosets that developed progressive neurologic symptoms within a practical time window as experimental animal models. In addition, it is notable that all second-generation transgenic offspring also carried the transgene with 120 CAG repeats, showing that the transgene was steadily transmitted to offspring.

In this study, two of three symptomatic transgenic marmosets underwent pathologic analyses and were found to have developed polyQ protein inclusions or degeneration in various regions, including brains, spinal cords, peripheral nerves, and muscles. This result is in line with previous studies in transgenic HD rhesus monkey models (Yang et al. 2008; Chan et al. 2015) that exhibited polyQ protein inclusions or degeneration in the cortex and striatum and suggests that the expression of mutant protein with expanded polyQ stretch resulted in these pathologic changes leading to abnormal motor phenotypes in both marmosets and rhesus monkeys. It is of note that the formation of inclusions composed of misfolded proteins and degeneration in the nervous system, which are key features shared among various neurodegenerative diseases such as AD, PD, and the polyQ diseases (Jucker and Walker, 2013; Nagai and Minakawa, 2015), were successfully reproducible in two different primate species.

Intriguingly, our marmoset model seems to have exhibited, at least in part, selectivity in the affected tissues similar to those in SCA3 patients, despite the widespread expression of ataxin 3-120Q protein under the CMV promoter. Indeed, the involvement of the cerebellum, the anterior horn of the spinal cord, and the peripheral nerves are often observed in SCA3 patients (Paulson, 2012). It is also known that the age of symptom onset in the juvenile form of SCA3 patients with a long 86 CAG repeat can be as early as 5 years (Zhou et al. 1997; Paulson, 2012), and that in SCA3, patients with homozygous expansions can be as young as 4 years old (Carvalho et al. 2008), resembling the relatively young onset of our transgenic marmoset line expressing hyperexpanded CAG repeats.

However, the strong ubiquitous expression of the mutant ataxin 3 protein via the CMV promoter could have resulted in the subacute disease progression of the motor phenotype and strong myopathic change in our marmosets, both of which are unusual in SCA3 patients. Furthermore, random integration effects of the lentiviral vector, including copy number variation, might have resulted in the variability of the behavioral and pathologic phenotype among our transgenic marmosets. Indeed, the fact that PQD2, which showed the earliest onset and rapid symp- 
tom progression, had a 2.8-fold higher transgene expression level compared with PQD6, which showed relatively late onset and slow symptom progression, suggests that the difference in the transgene expression level affected the severity of motor phenotypes in our marmosets, as was previously described in mouse models of SCA3 (Boy et al. 2009). Nevertheless, we used lentivirus-mediated gene transfer, because this has hitherto been the most successful method of producing transgenic nonhuman primates (Chan, 2013), although the establishment of more precise gene editing techniques that enables the generation of better NHP models such as gene knockouts/knockins is awaited for future studies.

Our transgenic marmoset model showing postgrowth disease onset and progressive motor impairment will be useful for various studies on neurodegenerative diseases, including the identification of disease biomarkers as well as the evaluation of the efficacy and metabolic profiles of therapeutic candidates (Morton and Howland, 2013; Howland and Munoz-Sanjuan, 2014). In addition, our MRI analyses suggested cerebellar atrophy in one of the disease-progressed living marmosets, illustrating a potential advantage of the use of nonhuman primate models with a larger brain size than rodents, which allows for more detailed in vivo anatomic evaluation (Hikishima et al. 2013). This advantage, when confirmed in the secondgeneration offspring obtained from our study, will allow us to monitor subtle temporal changes in the brain during disease onset and progression, or even before onset, by high-resolution functional brain imaging analyses such as $\mathrm{MRI}$ and PET. Although the symptomatic transgenic marmosets obtained in the first generation were limited in number and showed phenotypic variation, we successfully achieved transgenic offspring from the symptomatic transgenic marmoset. Despite the variation in the integration site in these offspring, possibly because of mosaicism in the founder marmoset, symptom onset in these offspring is anticipated considering the transgene expression comparable to that of the symptomatic founder marmosets in at least two of the four offspring, and the correlation of the transgene expression and symptom onset in the founder generation. We expect that the cohort of these symptomatic marmosets, once established from these offspring, can be used as a colony source and will contribute to future translational research in a timely manner, aid the development of clinically relevant biomarkers, and be used to evaluate the safety and efficacy of therapeutic candidates of polyQ diseases. Our success in modeling the polyQ diseases in common marmosets should accelerate research not only on neurodegenerative diseases, but also on various other human diseases (Chan, 2013; Kishi et al. 2014).

\section{References}

Boy J, Schmidt T, Wolburg H, Mack A, Nuber S, Bottcher M, Schmitt I, Holzmann C, Zimmermann F, Servadio A, Riess O (2009) Reversibility of symptoms in a conditional mouse model of spinocerebellar ataxia type 3. Hum Mol Genet 18:4282-4295. CrossRef Medline

Boy J, Schmidt T, Schumann U, Grasshoff U, Unser S, Holzmann C, Schmitt I, Karl T, Laccone F, Wolburg H, Ibrahim S, Riess O (2010)
A transgenic mouse model of spinocerebellar ataxia type 3 resembling late disease onset and gender-specific instability of CAG repeats. Neurobiol Dis 37:284-293. CrossRef Medline

Carvalho DR, La Rocque-Ferreira A, Rizzo IM, Imamura EU, SpeckMartins CE (2008) Homozygosity enhances severity in spinocerebellar ataxia type 3. Pediatr Neurol 38:296-299. CrossRef Medline Chan AW (2013) Progress and prospects for genetic modification of nonhuman primate models in biomedical research. ILAR J 54:211223. CrossRef Medline

Chan AW, Jiang J, Chen Y, Li C, Prucha MS, Hu Y, Chi T, Moran S, Rahim T, Li S, Li X, Zola SM, Testa CM, Mao H, Villalba R, Smith Y, Zhang X, Bachevalier J (2015) Progressive cognitive deficit, motor impairment and striatal pathology in a transgenic Huntington disease monkey model from infancy to adulthood. PLoS One 10:e0122335. CrossRef

Chan AW, et al. (2014) A two years longitudinal study of a transgenic Huntington disease monkey. BMC Neurosci 15:36. CrossRef Medline

Costa MC, Paulson HL (2012) Toward understanding MachadoJoseph disease. Prog Neurobiol 97:239-257. CrossRef Medline

Davies SW, Turmaine M, Cozens BA, DiFiglia M, Sharp AH, Ross CA, Scherzinger E, Wanker EE, Mangiarini L, Bates GP (1997) Formation of neuronal intranuclear inclusions underlies the neurological dysfunction in mice transgenic for the HD mutation. Cell 90:537548. CrossRef

Doody RS, Raman R, Farlow M, Iwatsubo T, Vellas B, Joffe S, Kieburtz K, He F, Sun X, Thomas RG, Aisen PS, Siemers E, Sethuraman G, Mohs R (2013) A phase 3 trial of semagacestat for treatment of Alzheimer's disease. N Engl J Med 369:341-350. CrossRef Medline

Doody RS, Thomas RG, Farlow M, Iwatsubo T, Vellas B, Joffe S, Kieburtz K, Raman R, Sun X, Aisen PS, Siemers E, Liu-Seifert H, Mohs R, Alzheimer's Disease Cooperative Study Steering Committee, Solanezumab Study Group (2014) Phase 3 trials of solanezumab for mild-to-moderate Alzheimer's disease. N Engl J Med 370:311-321. CrossRef

Hardman C, Ashwell K (2012) Stereotaxic and chemoarchitectural atlas of the brain of the common marmoset (Callithrix jacchus). CRC Press, Boca Raton, FL.

Hikishima K, Sawada K, Murayama AY, Komaki Y, Kawai K, Sato N, Inoue T, Itoh T, Momoshima S, Iriki A, Okano HJ, Sasaki E, Okano $\mathrm{H}$ (2013) Atlas of the developing brain of the marmoset monkey constructed using magnetic resonance histology. Neuroscience 230:102-113. CrossRef Medline

Howland DS, Munoz-Sanjuan I (2014) Mind the gap: models in multiple species needed for therapeutic development in Huntington's disease. Mov Disord 29:1397-1403. CrossRef Medline

Ishibashi $\mathrm{H}$, Motohashi HH, Kumon M, Yamamoto K, Okada $\mathrm{H}$, Okada T, Seki K (2013a) Ultrasound-guided non-surgical embryo collection in the common marmoset. Reprod Biol 13:139-144.

Ishibashi $\mathrm{H}$, Motohashi $\mathrm{HH}$, Kumon M, Yamamoto $\mathrm{K}$, Okada $\mathrm{H}$, Okada T, Seki K (2013b) Efficient embryo transfer in the common marmoset monkey (Callithrix jacchus) with a reduced transfer volume: a non-surgical approach with cryopreserved late-stage embryos. Biol Reprod 88:115.

Jucker M, Walker LC (2013) Self-propagation of pathogenic protein aggregates in neurodegenerative diseases. Nature 501:45-51. CrossRef Medline

Kishi N, Sato K, Sasaki E, Okano H (2014) Common marmoset as a new model animal for neuroscience research and genome editing technology. Dev Growth Differ 56:53-62. CrossRef Medline

Lenzmeier BA, Freudenreich CH (2003) Trinucleotide repeat instability: a hairpin curve at the crossroads of replication, recombination, and repair. Cytogenet Genome Res 100:7-24. Medline Medline

Malicdan MC, Noguchi S, Nishino I (2009) Monitoring autophagy in muscle diseases. Methods Enzymol 453:379-396. CrossRef Medline

Moran S, Chi T, Prucha MS, Ahn KS, Connor-Stroud F, Jean S, Gould K, Chan AW (2015) Germline transmission in transgenic 
Huntington's disease monkeys. Theriogenology 84:277-285. CrossRef Medline

Morton AJ, Howland DS (2013) Large genetic animal models of Huntington's Disease. J Huntingtons Dis 2:3-19. CrossRef Medline

Nagai Y, Popiel HA (2008) Conformational changes and aggregation of expanded polyglutamine proteins as therapeutic targets of the polyglutamine diseases: exposed beta-sheet hypothesis. Curr Pharm Des 14:3267-3279. Medline

Nagai Y, Minakawa EN (2015) Drug Development for Neurodegenerative Diseases. Springer, New York.

Paulson H (2012) Machado-Joseph disease/spinocerebellar ataxia type 3. Handb Clin Neurol 103:437-449. CrossRef Medline

Pinkel D, Straume T, Gray JW (1986) Cytogenetic analysis using quantitative, high-sensitivity, fluorescence hybridization. Proc Natl Acad Sci U S A 83:2934-2938. Medline

Rockenstein E, Crews L, Masliah E (2007) Transgenic animal models of neurodegenerative diseases and their application to treatment development. Adv Drug Deliv Rev 59:1093-1102. CrossRef Medline

Salloway S, et al. (2014) Two phase 3 trials of bapineuzumab in mild-to-moderate Alzheimer's disease. N Engl J Med 370:322333. CrossRef Medline

Sasaki E, Hanazawa K, Kurita R, Akatsuka A, Yoshizaki T, Ishii H, Tanioka Y, Ohnishi Y, Suemizu H, Sugawara A, Tamaoki N, Izawa K, Nakazaki Y, Hamada H, Suemori H, Asano S, Nakatsuji N, Okano H, Tani K (2005) Establishment of novel embryonic stem cell lines derived from the common marmoset (Callithrix jacchus). Stem Cells 23:1304-1313. CrossRef Medline

Sasaki E, et al. (2009) Generation of transgenic non-human primates with germline transmission. Nature 459:523-527. CrossRef Medline

Smith JP, Hicks PS, Ortiz LR, Martinez MJ, Mandler RN (1995) Quantitative measurement of muscle strength in the mouse. $J$ Neurosci Methods 62:15-19. Medline
Takahashi T, Hanazawa K, Inoue T, Sato K, Sedohara A, Okahara J, Suemizu H, Yagihashi C, Yamamoto M, Eto T, Konno Y, Okano H, Suematsu M, Sasaki E (2014) Birth of healthy offspring following ICSI in in vitro-matured common marmoset (Callithrix jacchus) oocytes. PLoS One 9:e95560 CrossRef Medline

Tomioka I, Takahashi T, Shimada A, Yoshioka K, Sasaki E (2012) Birth of common marmoset (Callithrix jacchus) offspring derived from in vitro-matured oocytes in chemically defined medium. Theriogenology 78:1487-1493. CrossRef Medline

Tomioka I, Maeda T, Shimada H, Kawai K, Okada Y, Igarashi H, Oiwa R, Iwasaki T, Aoki M, Kimura T, Shiozawa S, Shinohara H, Suemizu H, Sasaki E, Okano H (2010) Generating induced pluripotent stem cells from common marmoset (Callithrix jacchus) fetal liver cells using defined factors, including Lin28. Genes Cells 15:959-969. CrossRef Medline

Watase K, Weeber EJ, Xu B, Antalffy B, Yuva-Paylor L, Hashimoto K, Kano M, Atkinson R, Sun Y, Armstrong DL, Sweatt JD, Orr HT, Paylor R, Zoghbi HY (2002) A long CAG repeat in the mouse Sca1 locus replicates SCA1 features and reveals the impact of protein solubility on selective neurodegeneration. Neuron 34:905-919. Medline

Wong PC, Cai H, Borchelt DR, Price DL (2002) Genetically engineered mouse models of neurodegenerative diseases. Nat Neurosci 5:633-639. CrossRef Medline

Yang SH, Cheng PH, Banta $\mathrm{H}$, Piotrowska-Nitsche $\mathrm{K}$, Yang JJ, Cheng EC, Snyder B, Larkin K, Liu J, Orkin J, Fang ZH, Smith Y, Bachevalier J, Zola SM, Li SH, Li XJ, Chan AW (2008) Towards a transgenic model of Huntington's disease in a non-human primate. Nature 453:921-924. CrossRef

Zhou YX, Takiyama Y, Igarashi S, Li YF, Zhou BY, Gui DC, Endo K, Tanaka H, Chen ZH, Zhou LS, Fan MZ, Yang BX, Weissenbach J, Wang GX, Tsuji S (1997) Machado-Joseph disease in four Chinese pedigrees: molecular analysis of 15 patients including two juvenile cases and clinical correlations. Neurology 48:482-485. Medline 